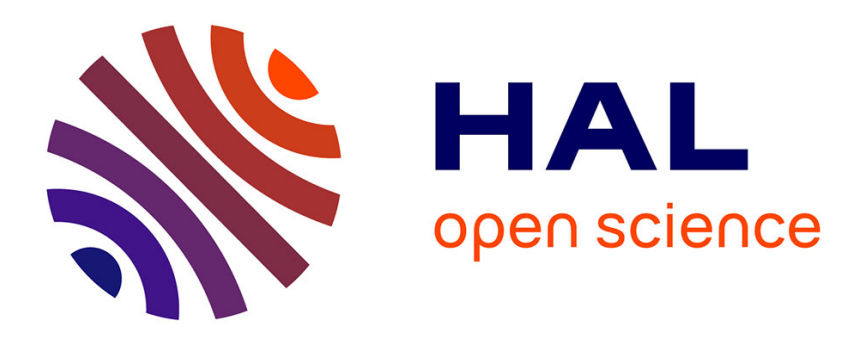

\title{
A Liouville theorem for a heat equation and applications for quenching
}

\author{
Nejla Nouaili
}

\section{To cite this version:}

Nejla Nouaili. A Liouville theorem for a heat equation and applications for quenching. Nonlinearity, 2011, 24 (3), pp.797-832. hal-00531228

\section{HAL Id: hal-00531228 \\ https://hal.science/hal-00531228}

Submitted on 2 Nov 2010

HAL is a multi-disciplinary open access archive for the deposit and dissemination of scientific research documents, whether they are published or not. The documents may come from teaching and research institutions in France or abroad, or from public or private research centers.
L'archive ouverte pluridisciplinaire HAL, est destinée au dépôt et à la diffusion de documents scientifiques de niveau recherche, publiés ou non, émanant des établissements d'enseignement et de recherche français ou étrangers, des laboratoires publics ou privés. 


\title{
A Liouville theorem for a heat equation and applications for quenching
}

\author{
Nejla Nouaili \\ Université Paris Dauphine
}

July 16, 2010

\begin{abstract}
We prove a Liouville Theorem for a semilinear heat equation with absorption term in one dimension. We then derive from this theorem uniform estimates for quenching solutions of that equation.
\end{abstract}

Mathematical Subject classification: 35K05, 35K55, 74H35.

Keywords: Quenching, Liouville theorem, heat equation.

\section{Introduction}

This paper is concerned with quenching solutions of the following nonlinear heat equation

$$
\left\{\begin{array}{l}
\partial_{t} u=\partial_{x}^{2} u-\frac{1}{u^{\beta}} \text { in } \mathbb{R} \times[0, T), \\
u(x, 0)=u_{0}(x)>0 \text { for } x \in \mathbb{R},
\end{array}\right.
$$

where $\beta \geq 3$ and

$$
u_{0}, \quad \frac{1}{u_{0}} \in L^{\infty}(\mathbb{R}) .
$$

We say that $u(t)$ quenches in finite time $T$ if $u$ exists for $t \in[0, T)$ and

$$
\lim _{t \rightarrow T} \inf _{x \in \mathbb{R}} u(x, t)=0 .
$$

Hereafter we consider a solution $u$ of (1) which quenches at finite time $T$. A point $a$ is said to be a quenching point if there is a sequence $\left\{\left(a_{n}, t_{n}\right)\right\}$ such that $a_{n} \rightarrow a, t_{n} \rightarrow T$ and $u\left(a_{n}, t_{n}\right) \rightarrow 0$ as $n \rightarrow \infty$.

Quenching phenomena play an important role and have many applications in physics, chemistry and biology ( see Salin [Sal04]). It may arise for instance in the theory of combustion and population genetics (see Galaktionov and Vázquez [GV02]), in population dynamics (see Gaucel and Langlais [GL07]), in connection with the diffusion equation generated by a polarization phenomena in ionic conductors (see Kawarada [Kaw75]), in connection with phase transition, when we study the motion of the borderline between liquids and solids (see Fila and Kawohl [FK92a]) and also in vortex reconnection with the 
boundary in type II superconductors (see Chapman and all [CHO98] and Merle and Zaag [MZ97]).

The study of the quenching problem (1) was initiated by Kawarada [Kaw75]. There is a considerable litterature on parabolic problems with strong absorption that deal with the precise description of the qualitative behavior of solutions near quenching. Typical questions in this respect concern the existence of quenching solutions as well as the quenching rates and profiles (see Deng and Levine [DL89], Fila and Kawohl [FK92a], Fila, Kawohl and Levine [FKBL92], Merle and Zaag [MZ97], Levine [Lev93] and Dávila and Montenegro [DM05]). Recently, equation (1) was numerically studied by Liang, Lin and Tan [LLT07].

The quenching rate as $t \rightarrow T$ of the solution $u$ of (1) near a quenching point is among the important issues. It was obtained by Guo on bounded domains [Guo90], [Guo91a], Fila and Kawohl [FK92a]. For the higher-dimensional radial problem in [Guo91b] and Fila, Kawohl and Quittner [FHQ92], under some restrictions on initial data.

In [Guo90], Guo showed that if

$$
u_{0}^{\prime \prime}-u_{0}^{-\beta} \leq 0,
$$

and $a$ is an arbitrary quenching point, then

$$
\lim _{t \rightarrow T} u(x, t)=U_{T}(t), \text { uniformly for }|x-a| \leq C(T-t)^{\frac{1}{2}},
$$

where $C>0$ and $U_{T}(t)=(\beta+1)^{\frac{1}{\beta+1}}(T-t)^{\frac{1}{\beta+1}}$ is the solution of $U_{T}^{\prime}=-\frac{1}{U_{T}^{\beta}}$ and $U_{T}(T)=0$.

In [FG93], Filippas and Guo were able to obtain a precise description of the profile of the solution in a neighborhood of the quenching point. They proved the following:

Let $u$ be a solution of (1) defined for $x \in(-1,1)$, with the boundary conditions $u( \pm 1, t)=1$, which quenches at the point a at time T. Moreover, we assume that $u_{0}^{\prime \prime}-\frac{1}{u_{0}^{\beta}} \leq 0$ and that $u_{0}$ has a single minimum. Then, we have that

$$
\left\{\begin{array}{l}
u(a, t) \rightarrow 0 \\
u(x, t) \rightarrow u^{*}(x) \text { as } t \rightarrow T \text { if } x \neq a \\
u^{*}(x)=\left[\frac{(\beta+1)^{2}}{8 \beta}\right]^{\frac{1}{(\beta+1)}}\left(\frac{|x-a|^{2}}{|\log | x-a||}\right)^{\frac{1}{(\beta+1)}}(1+o(1))
\end{array}\right.
$$

as $|x-a| \rightarrow 0$.

However, the result of Filippas and Guo is not uniform with respect to the quenching point or initial data. We aim in this paper at obtaining uniform estimates on $u(t)$ (defined on $\mathbb{R}$ ) at or near the singularity, that is as $t \rightarrow T$. In order to do so, we introduce for each $a \in \mathbb{R}$ the following similarity variables:

$$
y=\frac{x-a}{\sqrt{T-t}}, s=-\log (T-t), w_{a}(y, s)=(T-t)^{-\frac{1}{\beta+1}} u(x, t) .
$$

The function $w_{a}(=w)$ satisfies for all $s \geq-\log T$, and $y \in \mathbb{R}$ :

$$
\partial_{s} w=\partial_{y}^{2} w-\frac{1}{2} y \cdot \partial_{y} w+\frac{w}{\beta+1}-w^{-\beta},
$$


The study of $u(t)$ near $(a, T)$, where $a$ is a quenching point, is equivalent to the study of the long time behavior of $w_{a}$.

If we introduce $v$ defined by

$$
v=\frac{1}{u},
$$

then, we can see easily from (1) and (3), that $v$ satisfies the following equation:

$$
\partial_{t} v=\partial_{x}^{2} v-2 \frac{\left(\partial_{x} v\right)^{2}}{v}+v^{2+\beta} \text { in } \mathbb{R} \times[0, T),
$$

We note that $u$ quenches at time $T$ if and only if $v$ blows up at time $T$, and that $a$ is a quenching point for $u$ if and only if $a$ is a blow-up point for $v$.

As we did for $u$, we introduce for $v$ the following function:

$$
z_{a}(y, s)=(T-t)^{\frac{1}{\beta+1}} v(x, t), \text { where } y \text { and } s \text { are defined as in }(5) .
$$

Note from (5) and (7) that $z_{a}=\frac{1}{w_{a}}$. We get by (8) and (9), that $z_{a}(=z)$ satisfies for all $s \geq-\log T$, and $y \in \mathbb{R}$,

$$
\partial_{s} z=\partial_{y}^{2} z-\frac{1}{2} y \cdot \partial_{y} z-2 \frac{\left(\partial_{y} z\right)^{2}}{z}-\frac{z}{\beta+1}+z^{2+\beta} .
$$

We note by $L_{\rho}^{2}$ the Hilbert space defined by;

$$
L_{\rho}^{2}=\left\{g \in L_{l o c}^{2}\left(\mathbb{R}^{N}, \mathbb{C}\right), \int_{\mathbb{R}^{N}}|g|^{2} e^{-\frac{|y|^{2}}{4}} d y<+\infty\right\} \text { where } \rho(y)=\frac{e^{-\frac{|y|^{2}}{4}}}{(4 \pi)^{N / 2}} .
$$

If $g$ depends only on the variable $y \in \mathbb{R}^{N}$, we use the notation

$$
\|g\|_{L_{\rho}^{2}}^{2}=\int_{\mathbb{R}^{N}}|g(y)|^{2} e^{-\frac{|y|^{2}}{4}} d y .
$$

If $g$ depends only on $(y, s) \in \mathbb{R}^{N} \times \mathbb{R}$, we use the notation

$$
\|g(., s)\|_{L_{\rho}^{2}}^{2}=\int_{\mathbb{R}^{N}}|g(y, s)|^{2} e^{-\frac{|y|^{2}}{4}} d y .
$$

\subsection{A Liouville Theorem}

Our aim in this paper is to prove estimates for quenching solution of (6)), uniform with respect to the space variable $x$. The following Liouville theorem is crucial for that purpose.

Theorem 1 (A Liouville Theorem for equation (6)) Assume that $\beta \geq 3$. We consider $w$, a global nonnegative continuous solution of (6), satisfying

$$
\left|\partial_{y} w(y, s)\right|+\frac{1}{w(y, s)} \leq M, \text { for all }(y, s) \in \mathbb{R} \times \mathbb{R} \text {, where } M>0 .
$$


Then either

(i) $w \equiv \kappa$, or

(ii) there exists $s_{0} \in \mathbb{R}$ such that for all $(y, s) \in \mathbb{R} \times \mathbb{R}, w(y, s)=\varphi\left(s-s_{0}\right)$, where

$$
\varphi(s)=\kappa\left(1+e^{s}\right)^{\frac{1}{\beta+1}}
$$

is a solution of (6) independent of $y$ and satisfies

$$
\varphi^{\prime}=\frac{\varphi}{1+\beta}-\varphi^{-\beta}, \quad \varphi(-\infty)=\kappa, \quad \varphi(+\infty)=+\infty .
$$

Remark: If $0<\beta<3$, we don't have an explicit result for the global stationary solution of (6) as for the case $\beta>3$, the only result that we know is that there exist slow orbit, for more details see Guo [Guo91a], [Guo91b] and Fila, Kawohl and Quittner [FHQ92].

Theorem 1 has an equivalent formulation for solutions of equation (1):

Corollary 2 (A Liouville Theorem for equation (1)) Assume that $u$ is a nonnegative continuous solution of (1) defined for $(x, t) \in \mathbb{R} \times(-\infty, T)$. Assume in addition that $u(x, t) \geq B(T-t)^{\frac{1}{(\beta+1)}}$ for some $B>0$ and $\left\|\partial_{x} u(., t)\right\|_{L^{\infty}} \leq M(T-t)^{\frac{1}{\beta+1}-\frac{1}{2}}$, for $M>0$. Then, there exists $T_{0} \geq T$ such that for all $(x, t) \in \mathbb{R} \times(-\infty, T), u(x, T)=\kappa\left(T_{0}-t\right)^{\frac{1}{(\beta+1)}}$.

\section{$1.2 \quad$ Application to quenching}

We note that in the blow-up recent litterature ([MZ00], [MM00] and [MZ08]), Liouville Theorems have important applications to blow-up. In the quenching problem we can get similar results. In the following, we will say that a solution $u$ is of type $\mathbf{I}$, if it satisfy

$$
\left.\left\|\frac{1}{u(., t)}\right\|_{L^{\infty}(\mathbb{R})}(T-t)^{\frac{1}{\beta+1}}+\left\|\partial_{x} u(., t)\right\|_{L^{\infty}(\mathbb{R})}(T-t)^{-\frac{1}{\beta+1}+\frac{1}{2}} \leq M, \text { (type } \mathbf{I}\right)
$$

where $M$ is a positive constant.

Note that several authors proved the existence of type $\mathbf{I}$ solution (see for example Guo [Guo90] and [Guo91a]). We wander whether the bound on $\left\|\partial_{x} u\right\|_{L^{\infty}}$ in (14) follows from the bound on $\left\|\frac{1}{u}\right\|_{L^{\infty}}$. In other words, we wander whether we can charecterize the set of type $\mathbf{I}$ solution only by the fact that

$$
\left\|\frac{1}{u}\right\|_{L^{\infty}}(T-t)^{1 /(\beta+1)} \leq M \text { for some } M>0 .
$$

In the following, we derive from the Liouville Theorem some uniform estimates of type $\mathbf{I}$ quenching solutions of equation (6).

Theorem 3 i) (Uniform bounds on $u(t)$ on quenching time) Assume that $u(t)$ is a nonnegative solution of equation (1) that quenches in finite time $T>0$ with initial data $u_{0}$ satisfying (2). Then, we have

$$
\inf _{x \in \mathbb{R}} u(x, t) \sim U_{T}(t)=(\beta+1)^{\frac{1}{\beta+1}}(T-t)^{\frac{1}{\beta+1}} \text { as } t \rightarrow T,
$$


and

$$
(T-t)^{\frac{1}{\beta+1}+\frac{1}{2}}\left\|\partial_{x} v(., t)\right\|_{L^{\infty}(\mathbb{R})}+(T-t)^{\frac{1}{\beta+1}+1}\left\|\partial_{x}^{2} v(., t)\right\|_{L^{\infty}(\mathbb{R})} \rightarrow 0 \text { as } t \rightarrow T .
$$

Equivalently, for any $a \in \mathbb{R}$,

$$
\inf _{y \in \mathbb{R}} w_{a}(y, s) \rightarrow \kappa \text { as } s \rightarrow+\infty \text { and }\left\|\partial_{y} z_{a}(., s)\right\|_{L^{\infty}}+\left\|\partial_{y}^{2} z_{a}(., s)\right\|_{L^{\infty}} \rightarrow 0 \text { as } s \rightarrow+\infty
$$

where $\kappa=(1+\beta)^{\frac{1}{\beta+1}}$.

ii) (ODE-type behavior) For all $\varepsilon>0$, there exists $C_{\varepsilon}>0$, such that, for all $t \in\left[\frac{T}{2}, T\right)$ and $x \in \mathbb{R}$,

$$
\left|\frac{\partial u}{\partial t}-u^{-\beta}\right| \leq \varepsilon|u|^{-\beta}+C_{\varepsilon}
$$

\subsection{Strategy of the proof of the Liouville theorem}

Our method is inspired by the one of Merle and Zaag [MZ98a] and [MZ00] developed for the semilinear heat equation

$$
\partial_{t} u=\Delta u+|u|^{p-1} u
$$

where

$$
u:(x, t) \in \mathbb{R}^{N} \times[0, T) \rightarrow \mathbb{R} \text { and } p>1, \quad p<\frac{N+2}{N-2} \text { if } N \geq 3 .
$$

However, our contribution is not a simple adaptation of the proof of [MZ98a] and [MZ00]. In fact, in these papers the authors strongly rely on two blow-up criteria for the selfsimilar version of $(16)$

$$
w_{s}=\Delta w-\frac{1}{2} y \partial_{y} w-\frac{w}{p-1}+|w|^{p-1} w .
$$

- Criterion 1: For nonnegative solution of (17) such that

$$
\int_{\mathbb{R}^{N}} w\left(y, s_{0}\right) \rho(y) d y>(p-1)^{\frac{1}{p-1}},
$$

for some $s_{0} \in \mathbb{R}$, then $w$ blows up in some finite time $s>s_{0}$.

- Criterion 2: (with no sign condition) Assume that $w$ is a solution of (17) such that

$$
\mathcal{E}\left(w\left(s_{0}\right)\right) \leq \frac{(p-1)}{2(p+1)}\left(\int_{\mathbb{R}^{N}}\left|w\left(y, s_{0}\right)\right|^{2} \rho(y) d y\right)^{\frac{p+1}{2}},
$$

for some $s_{0} \in \mathbb{R}$, where $\mathcal{E}$ is the following Lyapunov functional defined by

$$
\mathcal{E}(w)=\int_{\mathbb{R}}\left(\frac{1}{2}\left|\partial_{y} w\right|^{2}+\frac{|w|^{2}}{2(p-1)}-\frac{|w|^{p+1}}{p+1}\right) \rho(y) d y \text { with } \rho(y)=\frac{e^{-\frac{|y|^{2}}{4}}}{(4 \pi)^{N / 2}} .
$$

Then, $w$ blows up in finite time $s>s_{0}$. 
Note that in [Zaa01], Zaag obtained a Liouville theorem for the non-gradient structure system

$$
\begin{cases}\partial_{t} u=\Delta u+v^{p}, & \partial_{t} v=\Delta v+u^{q} \\ u(., 0)=u_{0}, & v(., 0)=v_{0}\end{cases}
$$

where $p-q$ is small. There, he adapts the proof of [MZ98a] and uses an infinite time blow-up criterion in similarity variables.

In our quenching problem, there is no way to find any equivalent blow-up criterion. We need new ideas which make the originality of our paper. From this point of view the difficulty level is similar to the case of the complex valued equation with no gradient structure

$$
\partial_{t} u=\Delta u+(1+i \delta)|u|^{p-1} u
$$

where $u: \mathbb{R}^{N} \times \mathbb{R} \rightarrow \mathbb{C}$.

treated in [NZ10]. We proceed in five parts.

- Part 1 , we show that $z(=1 / w)$ has a limit $z_{ \pm \infty}$ as $s \rightarrow \pm \infty$, where $z_{ \pm \infty}$ is a critical point of the stationary version of (10) or 0 . That is, $z_{ \pm \infty} \equiv \kappa^{-1}$ or $z_{ \pm \infty} \equiv 0$, thanks to Guo. Then, we rule out the case where $z_{-\infty} \equiv 0$ with sophisticated energy argument. The following parts are dedicated to the non trivial case where $z_{-\infty}=\kappa^{-1}$.

- in Part 2, we investigate the linear problem (in $w$ ) around $\kappa$ as $s \rightarrow-\infty$ and show that $w$ behaves at most in three ways:

- (i) $w(y, s)=\kappa+C_{0} e^{s}+o\left(e^{s}\right)$ as $s \rightarrow-\infty$, for some constant $C_{0} \in \mathbb{R}$.

- (ii) $w(y, s)=\kappa+C_{1} e^{s / 2}+o\left(e^{s / 2}\right)$ as $s \rightarrow-\infty$, for some constant $C_{1} \in \mathbb{R} \backslash\{0\}$.

- (iii) $w(y, s)=\kappa+\frac{\kappa}{2 \beta s}\left(\frac{1}{2} y^{2}-1\right)+o\left(\frac{1}{s}\right)$ as $s \rightarrow-\infty$.

In these cases, convergence take place in $L^{\infty}([-R, R])$ for any $R>0$ and in $L_{\rho}^{2}$.

- In Part 3, we show that (i) corresponds to $w(y, s)=\varphi\left(s-s_{0}\right)$ or $w(y, s)=\kappa$, for some $s_{0} \in \mathbb{R}$, where $\varphi$ is defined by (13).

- In Parts 4 and 5, we rule out cases (ii) and (iii). In [MZ98a] and [MZ00], the authors shows that for some $a_{0} \in \mathbb{R}$ and $s_{0} \in \mathbb{R}, w_{a_{0}}(y, s)=w\left(y+a_{0} e^{s_{0}}, s_{0}\right)$ satisfies one of the blow-up criteria stated in page (5), which contradicts the fact that $w$ exists for all $s \in \mathbb{R}$. In our case we don't have any blow-up criterion. It turns out that this is the major difficulty in our paper, as in [NZ10] for equation (19). Following [NZ10], we will use a geometrical method where the key idea is to extend the convergence stated in (ii) and (iii) from compact sets to larger zones, so that we find the singular profile for $w$. It appears that in both cases, for larger $|y|$, this profile becomes strictly inferior to $\frac{1}{M}$, where $M$ is defined in (11), which is a contradiction. The originality of our paper is based on Velázquez's work in [Vel92], where he extends the convergence from compact sets to larger sets to find the profile for solutions of (17). Note that the fact that $w$ is not in $L^{\infty}$ makes it delicate to use the estimate of [Vel92]. More precisely, we obtain the following singular profiles 
- If case (ii) holds, then

$$
\lim _{s \rightarrow-\infty} \sup _{|y| \leq R e^{-s / 2}}\left|w(y, s)-\left((\beta+1)-C_{1} \kappa^{-\beta} y e^{s / 2}\right)^{\frac{1}{\beta+1}}\right|=0
$$

with $0<R<\frac{(\beta+1)}{C_{1} \kappa^{-\beta}}$ and $C_{1} \neq 0$.

- If case (iii) holds, then

$$
\lim _{s \rightarrow-\infty} \sup _{|y| \leq \sqrt{-s} R}\left|w(y, s)-\left((\beta+1)+\frac{(\beta+1)^{2}}{4 \beta} \frac{y^{2}}{s}\right)^{\frac{1}{\beta+1}}\right|
$$

with $0<R<\sqrt{\frac{4 \beta}{(\beta+1)}}$.

Then using condition (11), case (ii) and (iii) are ruled out, which ends the proof of our Liouville Theorem.

Our paper is organised as follows:

In section 2, we prove the Liouville theorem.

In section 3, we prove the uniform estimates for quenching solutions of (6) in Theorem 3.

\section{Proof of the Liouville Theorem for equation (6)}

We assume that $\beta \geq 3$ and consider $w(y, s)$ a nonnegative, global solution of (6) satisfying (11), defined for all $(y, s) \in \mathbb{R} \times \mathbb{R}$. Introducing $z=\frac{1}{w}$, we know that $z$ satisfies (10) on $\mathbb{R}^{2}$.

Our goal is to show that $w$ depends only on the variable $s$.

\subsection{Part 1: Behavior of $w$ as $s \rightarrow \pm \infty$}

The main results of this part are consequences of the parabolic estimates and the gradient structure of equation (6). Let us recall them.

Lemma 2.1 (Parabolic estimates) There exists $M_{0}>0$ such that for all $(y, s) \in \mathbb{R} \times \mathbb{R}$ :

(i) $\left|\partial_{y} w(y, s)\right|+\frac{1}{w(y, s)} \leq M_{0}$ and $w(y, s) \leq w(0, s)+M_{0}|y|$,

(ii) $|z(y, s)|+\left|\partial_{y} z(y, s)\right|+\left|\partial_{y}^{2} z(y, s)\right| \leq M_{0}$ and $\left|\partial_{s} z\right| \leq M_{0}(1+|y|)$.

(iii) For all $R>0, z, \partial_{y} z(y, s), \partial_{y}^{2} z$ and $\partial_{s} z$ are bounded in $C_{\alpha}^{0}\left([-R, R]^{2}\right)$ for some $\alpha \in(0,1)$, where

$$
C_{\alpha}^{0}\left([-R, R]^{2}\right)=\left\{\psi \in L^{\infty}\left[(-R, R)^{2}\right] \mid \sup _{(\xi, \tau),\left(\xi^{\prime}, \tau^{\prime}\right) \in[-R, R]^{2}} \frac{\left|\psi(\xi, \tau)-\psi\left(\xi^{\prime}, \tau^{\prime}\right)\right|}{\left(\left|\xi-\xi^{\prime}\right|+\left|\tau-\tau^{\prime}\right|^{1 / 2}\right)^{\alpha}}<\infty\right\}
$$

(iv) $\left|\partial_{s} w(y, s)\right| \leq M_{0}(1+|y|)(w(0, s)+|y|)^{2}$. 
Proof:

(i) See (11).

(ii)and (iii) See the proof of Lemma 3.2 from [Guo90].

(iv) If we write $\partial_{s} w=-\partial_{s} z / z^{2}=-\partial_{s} z w^{2}$, then we get the result by (i) and (ii).

Lemma 2.2 (Gradient Structure)

(i) (Gradient Structure for equation (6)). If we introduce

$$
\boldsymbol{E}(w(s))=\int_{\mathbb{R}} \partial_{y} w^{2} \rho d y-\frac{1}{2(\beta+1)} \int_{\mathbb{R}} w^{2} \rho d y-\frac{1}{\beta-1} \int_{\mathbb{R}} w^{-(\beta-1)} \rho d y \text { with } \rho(y)=\frac{e^{-\frac{y^{2}}{4}}}{(4 \pi)^{\frac{1}{2}}},
$$

then, for all $s_{1}<s_{2} \in \mathbb{R}$, such that

$$
0<w(y, s) \leq c_{1}(s)(1+|y|) \text { for all } s_{1} \leq s \leq s_{2}\left(\text { with } c_{1}(s)>0\right),
$$

we have

$$
\int_{s_{1}}^{s_{2}} \int_{\mathbb{R}}\left(\partial_{s} w\right)^{2} \rho d y=\boldsymbol{E}\left(w\left(s_{1}\right)\right)-\boldsymbol{E}\left(w\left(s_{2}\right)\right)
$$

(ii) (Gradient Structure for equation (10)).If we define for each z solution of (10)

$$
E(z(s))=\int_{\mathbb{R}} \frac{\partial_{y} z^{2}}{z^{4}} \rho d y-\frac{1}{2(\beta+1)} \int_{\mathbb{R}} \frac{1}{z^{2}} \rho d y-\frac{1}{\beta-1} \int_{\mathbb{R}} z^{\beta-1} \rho d y,
$$

under the same conditions in (i), we obtain:

$$
\int_{a}^{b} \int_{\mathbb{R}}\left(\frac{\partial_{s} z}{z^{2}}\right)^{2} \rho d y=E(z(a))-E(z(b)), \text { for any real } a<b .
$$

Proof:

(i) One may multiply equation (10) by $\partial_{s} w \rho$ and integrate over the ball $B_{R}=B(O, R)$ with $R>0$. Then using Lemma 2.1 and the Lebesgue's theorem yields the result. (see Proposition 3 from Giga and Kohn [GK85] for more details).

(ii) Since $E(z)=\mathbf{E}\left(\frac{1}{z}\right)$, this immediately follows from (i)

We now give the limits of $z$ as $s \rightarrow \pm \infty$ in the following:

Proposition 2.3 (Limit of $z$ as $s \rightarrow \pm \infty$ ) The limit $z_{+\infty}(y)=\lim _{s \rightarrow+\infty} z(y, s)$ exists and equals 0 or $\kappa^{-1}$. The convergence is uniform on every compact subset of $\mathbb{R}$. The corresponding statements also hold for the limit $z_{-\infty}(y)=\lim _{s \rightarrow-\infty} z(y, s)$.

Proof: It follows from the following:

Lemma 2.4 Consider any increasing (respectively decreasing) sequence such that $s_{j} \rightarrow$ $\pm \infty$ as $j \rightarrow \infty$. Then:

(i) there is a subsequence (still denoted by $\left(s_{j}\right)$ ) such that

$$
z\left(y, s+s_{j}\right) \rightarrow l \text { in } C_{\alpha}^{2}([-R, R]) \text { for all } R>0 \text {, where } l=0 \text { or } \kappa^{-1} .
$$

(ii) If $l=0$, then $E\left(z\left(s_{j}\right)\right) \rightarrow-\infty$, if $l=\kappa^{-1}$, then $E\left(z\left(s_{j}\right)\right) \rightarrow E\left(\kappa^{-1}\right)$. 
Indeed, from Lemma 2.4, in order to get the conclusion of Proposition 2.3, it is enough to show that the limit in Lemma 2.4 is independent of the choice of the sequence. We consider the case $s \rightarrow \infty$, the other case being similar. Suppose that $\left(s_{j}\right)$ and $\left(\bar{s}_{j}\right)$ both tend to infinity. Up to extracting subsequence, we assume that for all $j \in \mathbb{N}, \quad s_{j}>\bar{s}_{j}$ and proceeding by contradiction, we assume that

$$
z_{j}(y, s)=z\left(y, s+s_{j}\right) \rightarrow \kappa^{-1} \text { and } \bar{z}_{j}(y, s)=z\left(y, s+\overline{s_{j}}\right) \rightarrow 0 \text { as } j \rightarrow \infty .
$$

By (ii) of Lemma 2.4, we have

$$
E\left(z_{j}(s)\right) \rightarrow E\left(\kappa^{-1}\right) \text { and } E\left(\bar{z}_{j}(s)\right) \rightarrow-\infty,
$$

hence, for $j$ large enough, we have

$$
s_{j}>\bar{s}_{j}, E\left(z\left(s_{j}\right)\right)>E\left(\kappa^{-1}\right)-1>E\left(z\left(\bar{s}_{j}\right)\right),
$$

which contradicts the monotonicity of $E$. Thus the limit in Lemma 2.4 is independent of the choice of the sequence and the whole function $z(y, s)$ converges as $s \rightarrow \infty$. It remains to prove Lemma 2.4 to finish the proof.

\section{Proof of Lemma 2.4:}

(i) We only present the case $s_{j} \rightarrow+\infty$, the analysis for $s_{j} \rightarrow-\infty$ is the same.

Let us recall that for all $(y, s), z(y, s)$ satisfies $(10)$, which is:

$$
\partial_{s} z=\partial_{y}^{2} z-\frac{1}{2} y \partial_{y} z-2 \frac{\partial_{y} z^{2}}{z}-\frac{z}{\beta+1}+z^{2+\beta}
$$

From condition (11), we get $0 \leq \frac{\left(\partial_{y} z\right)^{2}}{z}=\frac{\left(\partial_{y} w\right)^{2}}{w^{2}} z \leq C z$ and $0 \leq z^{2+\beta} \leq C z$, hence we obtain:

$$
\mathcal{L}_{0} z-C z \leq \partial_{s} z \leq \mathcal{L}_{0} z+C z \text { where } \mathcal{L}_{0} z=\partial_{y}^{2} z-\frac{1}{2} y \partial_{y} z .
$$

Using the semigroup $S_{0}(\tau)$ associated to $\mathcal{L}_{0}$ :

$$
S_{0}(\tau) \phi(y)=\frac{1}{\left(4 \pi\left(1-e^{-\tau}\right)\right)^{1 / 2}} \int_{\mathbb{R}} \exp \left(-\frac{\left(y e^{-\frac{\tau}{2}}-\lambda\right)}{4\left(1-e^{-\tau}\right)}\right) \phi(\lambda) d \lambda,
$$

we see that for all $s \geq s^{\prime}$,

$$
e^{-C\left(s-s^{\prime}\right)} S_{0}\left(s-s^{\prime}\right) z\left(., s^{\prime}\right) \leq\|z(., s)\|_{L^{\infty}} \leq e^{C\left(s-s^{\prime}\right)}\left\|z\left(., s^{\prime}\right)\right\|_{L^{\infty}} .
$$

Let $\left(s_{j}\right)$ be a sequence tending to $+\infty$, and let $z_{j}(y, s)=z\left(y, s+s_{j}\right)$.

From Lemma 2.1, up to extracting a sub-sequence (still denoted by $\left(s_{j}\right)$ ), $z_{j}$ converges to $\tilde{z}_{+\infty}$ in $C^{2,1}\left((-R, R)^{2}\right)$ for any $R>0$ and we obtain that $\tilde{z}_{+\infty}$ satisfy $(25)$.

In the following, we will prove that the limit $\tilde{z}_{+\infty}$ is either 0 or $\kappa^{-1}$. We have to consider two cases. .

Case 1: There exists $\left(y_{0}, s_{0}\right) \in \mathbb{R}^{2}$ such that $\tilde{z}_{+\infty}\left(y_{0}, s_{0}\right)=0$

We claim first that

$$
\forall y \in \mathbb{R}, \quad \tilde{z}_{+\infty}\left(y, s_{0}\right)=0 .
$$


Indeed, if for some $y_{1} \in \mathbb{R}$, we have $\tilde{z}_{+\infty}\left(y_{1}, s_{0}\right)>0$, then since we have from (11)

$$
\frac{1}{z_{j}\left(y_{0}, s_{0}\right)} \leq \frac{1}{z_{j}\left(y_{1}, s_{0}\right)}+M\left|y_{1}-y_{0}\right|
$$

letting $j \rightarrow \infty$, we get a contradiction. Thus (28) holds. Using (27), we conclude that $z \equiv 0$ on $\mathbb{R}^{2}$ (indeed, by (27) for $s \geq s_{0}, z(., s) \equiv 0$, and for $s \leq s_{0}, S_{0}\left(s_{0}-s\right) z(., s) \equiv 0$, hence $z(., s) \equiv 0)$.

Case 2: For all $(y, s) \in \mathbb{R}^{2}, \tilde{z}_{+\infty}(y, s)>0$

Let us introduce $w_{j}(y, s)=w\left(y, s+s_{j}\right)$. In this case,

$$
w_{j}(y, s)=1 / z_{j}(y, s) \rightarrow \tilde{w}_{+\infty}=1 / \tilde{z}_{+\infty}(y, s) \text { in } C^{2,1}\left((-R, R)^{2}\right), \text { for any } R>0 .
$$

Since $z_{j}$ and $w_{j}$ are solutions of (24) and (6) respectively, the same holds for $\tilde{z}_{+\infty}$ and $\tilde{w}_{+\infty}$ respectively. Our goal in this step is to prove that $\tilde{z}_{+\infty}(y, s)=\kappa^{-1}$. First, we prove that $\tilde{z}_{+\infty}$ is independent of $s$, then we conclude using the result of Guo concerning the stationary global solution of (6). We will proceed as in Proposition 4, page 308 in [GK85]. We claim the following:

Claim 2.5 (i) For all $(y, s) \in \mathbb{R}^{2}$

$$
\begin{aligned}
& \left|\partial_{y} w_{j}(y, s)\right|+\left|1 / w_{j}(., s)\right| \leq C_{1}, \quad\left|w_{j}(y, s)\right| \leq C_{1}\left(|y|+\tilde{w}_{+\infty}(0, s)\right) \\
& \quad \text { and }\left|\partial_{s} w_{j}(y, s)\right| \leq C_{1}(1+|y|)\left(|y|+\tilde{w}_{+\infty}(0, s)\right)^{2}
\end{aligned}
$$

where $C_{1}>0$ independent of $j$ and $\tilde{w}_{+\infty}$ is a solution of (6).

(ii) For all $(y, s) \in \mathbb{R}^{2}$,

$$
\begin{aligned}
& \left|\partial_{y} \tilde{w}_{+\infty}(y, s)\right|+\left|1 / \tilde{w}_{+\infty}(y, s)\right| \leq C_{1}, \quad\left|\tilde{w}_{+\infty}(y, s)\right| \leq C_{1}\left(|y|+\tilde{w}_{+\infty}(0, s)\right) \\
& \text { and }\left|\partial_{s} \tilde{w}_{\infty}(y, s)\right| \leq C_{1}(1+|y|)\left(|y|+\tilde{w}_{+\infty}(0, s)\right)^{2} .
\end{aligned}
$$

Remark: To make the notation legible, we will note the partial derivative in time and space of the sequence $w_{j}$ (or $z_{j}$ ) and the limit $\tilde{w}_{+\infty}\left(\right.$ or $\left.\tilde{z}_{+\infty}\right)$ respectively $\partial_{s} w_{j}, \partial_{y} w_{j}$ and $\partial_{s} \tilde{w}_{+\infty}, \partial_{y} \tilde{w}_{+\infty}$.

Proof of Claim 2.5: (i) Integrating the inequality $\left|\partial_{y} w_{j}(y, s)\right| \leq M$ (by (i) of Lemma 2.1) in space between 0 and $y$, we obtain $\left|w_{j}(y, s)\right| \leq M|y|+w_{j}(0, s)$. Then using the fact that

$$
w_{j}(0, s) \rightarrow \tilde{w}_{+\infty}(0, s)\left(=1 / \tilde{z}_{+\infty}(0, s)\right)<+\infty \text {, as } j \rightarrow \infty,
$$

we get the second inequality of (i) in Claim (2.5). Using Lemma 2.1 and the identity

$$
\partial_{s} w_{j}(y, s)=-\partial_{s} z_{j}(y, s) / z_{j}(y, s)^{2}=-\partial_{s} z_{j}(y, s) w_{j}(y, s)^{2},
$$

we obtain the third inequality in (i).

(ii) Using (i) and the convergence (29), the result comes immediately.

Up to extracting a subsequence, we can assume that $s_{j+1}-s_{j} \rightarrow \infty$. Therefore, using (21) with $w=w_{j}, a=m, b=m+s_{j+1}-s_{j}$, where $m \in \mathbb{Z}$, we get: 


$$
\begin{aligned}
\int_{m}^{s_{i+1}-s_{j}+m} \int_{\mathbb{R}}\left(\partial_{s} w_{j}\right)^{2} \rho d y & =\mathbf{E}\left(w_{j}(m)\right)-\mathbf{E}\left(w_{j}\left(m+s_{j+1}-s_{j}\right)\right) \\
& =\mathbf{E}\left(w_{j}(m)\right)-\mathbf{E}\left(w_{j+1}(m)\right) .
\end{aligned}
$$

Using (i), (ii) and (iii) of Claim (2.5) together with Lesbegue's Theorem we have

$$
\begin{array}{ll}
\int_{\mathbb{R}} \partial_{y} w_{j}^{2} \rho d y \rightarrow \int_{\mathbb{R}} \partial_{y} \tilde{w}_{+\infty}^{2} \rho d y, & \int_{\mathbb{R}} w_{j}^{2} \rho d y \rightarrow \int_{\mathbb{R}} \tilde{w}_{+\infty}^{2} \rho d y, \\
\text { and } \int_{\mathbb{R}} w_{j}^{-(\beta-1)} \rho d y \rightarrow \int_{\mathbb{R}} \tilde{w}_{+\infty}^{-(\beta-1)} \rho d y & \text { as } j \rightarrow \infty .
\end{array}
$$

Therefore, $\mathbf{E}\left(w_{j}(m)\right) \rightarrow \mathbf{E}\left(\tilde{w}_{+\infty}(m)\right)$ as $j \rightarrow \infty$ (well defined by (iii) of Claim 2.5). In particular, the right-hand side of (31) tends to zero. Since $s_{j+1}-s_{j} \rightarrow+\infty$, it follows that for every integers $m<M$

$$
\text { on the one hand } \lim _{j \rightarrow \infty} \int_{m}^{M} \int_{\mathbb{R}}\left|\partial_{s} w_{j}\right|^{2} \rho d y d s=0,
$$

On the other hand, by the third inequality in (i) of Claim 2.5 and the continuity of $\tilde{w}_{+\infty}(0, s)$, we obtain

$$
\forall(y, s) \in \mathbb{R} \times(m, M) \quad\left|\partial_{s} w_{j}(y, s)\right| \leq C(m, M)(1+|y|)^{3} .
$$

Since $\partial_{s} w_{j}$ converges simply to $\partial_{s} \tilde{w}_{+\infty}$ by (iii) of Claim 2.5 , we conclude that:

$$
\int_{m}^{M} \int_{\mathbb{R}}\left|\partial_{s} w_{j}\right|^{2} \rho d y d s \rightarrow \int_{m}^{M} \int_{\mathbb{R}}\left|\partial_{s} \tilde{w}_{+\infty}\right|^{2} \rho d y d s
$$

Using (32) and (33), we get

$$
\int_{m}^{M} \int_{\mathbb{R}}\left|\partial_{s} \tilde{w}_{+\infty}\right|^{2} \rho d y d s=0
$$

Using the fact that $m$ and $M$ are arbitrary, we conclude that $\partial_{s} \tilde{w}_{+\infty}=0$ on $\mathbb{R}^{2}$ and $\tilde{w}_{+\infty}(y, s)=\tilde{w}_{+\infty}(y)$ is independent of $s$. Since $\tilde{w}_{+\infty}$ is a solution of $(6)$, it follows that $\tilde{w}_{+\infty}$ solves the following stationary equation

$$
\forall y \in \mathbb{R}, \quad 0=w^{\prime \prime}-\frac{1}{2} y w^{\prime}+\frac{1}{\beta+1} w-w^{-\beta} .
$$

We recall that by (ii) of Claim 2.5 we have $\tilde{w}_{+\infty}(s)>1 / C$ and $\tilde{w}_{+\infty}(s) \leq C(1+|y|)$. Now we recall the result of Guo gived by Theorem 2.1 in [Guo90]):

The only global solution of

$$
w^{\prime \prime}-\frac{1}{2} y w^{\prime}+\frac{w}{\beta+1}-\varepsilon w^{-\beta}=0, y \in \mathbb{R}
$$

which is greater than or equal to some positive constant $c$ and which grows at most polynomially as $|y| \rightarrow \infty$, is $w \equiv(\varepsilon(\beta+1))^{\frac{1}{\beta+1}}$. 
Using this result, it follows that $\tilde{w}_{+\infty}=\kappa$ and we conclude that $\tilde{z}_{+\infty} \equiv \kappa^{-1}$. This concludes the proof of (i) of Lemma 2.4.

(ii) We recall from Lemma 2.2 that

$$
E(z(s))=\int_{\mathbb{R}} \frac{\partial_{y} z^{2}}{z^{4}} \rho d y-\frac{1}{2(\beta+1)} \int_{\mathbb{R}} \frac{1}{z^{2}} \rho d y-\frac{1}{\beta-1} \int_{\mathbb{R}} z^{\beta-1} \rho d y .
$$

If $z_{j}$ converges to $\kappa^{-1}$, using Lesbegue's Theorem and Claim 2.5, we obtain

$$
E\left(z_{j}(s)\right)=\mathbf{E}\left(w_{j}(s)\right) \rightarrow E\left(\kappa^{-1}\right)=\mathbf{E}(\kappa) .
$$

In the case, where $z_{j}$ converges to 0 , we write from Lemma 2.2 and Claim 2.5

$$
E\left(z_{j}(s)\right)=\mathbf{E}\left(w_{j}(s)\right) \leq C-\frac{1}{\beta-1} \int w^{-(\beta-1)} \rho d y
$$

Since the integral in (36) tends to infinity as $j \rightarrow+\infty$ we have:

$$
E\left(z_{j}(s)\right) \rightarrow-\infty
$$

This concludes the proof of Lemma 2.4 and Proposition 2.3 .

To end this part, we recall the result obtained by Proposition 2.3, which says that $z_{+\infty}(y)=\lim _{s \rightarrow+\infty} z(y, s)$ and $z_{-\infty}(y)=\lim _{s \rightarrow-\infty} z(y, s)$ exist and equal 0 or $\kappa^{-1}$. Now, we will prove that the case $z_{-\infty}=0$ can't occur.

Indeed, suppose that $z(y, s) \rightarrow 0$ as $s \rightarrow-\infty$, by (ii) of Lemma 2.4, we have:

$$
E(z(s)) \rightarrow-\infty \text { as } s \rightarrow-\infty
$$

Therefore, there exists $s_{1}<0$, such that for all $s \leq s_{1}$, we have

$$
s<0 \text { and } E(z(s))<E(z(0)) \text {. }
$$

which contradicts the monotonicity of $E$. Thus, the case $z_{-\infty}=0$ is ruled out. In the following, we will study the case where $w_{-\infty}=\kappa$.

\subsection{Part 2: Linear behavior of $w$ near $\kappa$ as $s \rightarrow-\infty$}

In this part, we assume that

$$
w \rightarrow \kappa \text { as } s \rightarrow-\infty
$$

in $L_{\rho}^{2}$ and uniformly on every compact sets, and we classify the $L_{\rho}^{2}$ behavior of $w-\kappa$ as $s \rightarrow-\infty$. Let us introduce $v=w-\kappa$. From (6), $v$ satisfies the following equation:

$$
\forall(y, s) \in \mathbb{R}^{2}, \partial_{s} v=\mathcal{L} v-f(v),
$$

where

$$
\begin{aligned}
& \mathcal{L} v=\partial_{y}^{2} v-\frac{1}{2} y \partial_{y} v+v \text { and } \\
& f(v)=(v+\kappa)^{-\beta}-\kappa^{-\beta}+\beta \kappa^{-(\beta+1)} v .
\end{aligned}
$$

Concerning the non-linear term of equation (39), we have: 
Lemma 2.6 There exists $s_{1} \in \mathbb{R}$ such that for all $s \leq s_{1}$ :

$$
0 \leq f(v) \leq M_{1} v^{2} \text { and } f(v) \leq M_{2}|v| .
$$

Proof: Using Taylor's formula, we obtain

$$
(v+\kappa)^{-\beta}=\kappa^{-\beta}-\frac{\beta}{(\beta+1)} v+\frac{1}{2} \beta(\beta+1)(\theta+\kappa)^{-\beta-2} v^{2},
$$

for some $\theta$ between 0 and $v$. Therefore

$$
f(v)=c(\theta, \beta) v^{2}, \quad c(\theta, \beta)=\frac{1}{2} \beta(\beta+1)(\theta+\kappa)^{-\beta-2} .
$$

If $v>0$ then $0 \leq c(\theta, \beta) \leq(\beta / 2)(\beta+1) \kappa^{-\beta-2}=\beta /(2 \kappa)$.

If $v<0$, recalling that $B \leq v+\kappa \leq \theta+\kappa$ we obtain $0 \leq c(\theta, \beta) \leq(\beta / 2)(\beta+1) B^{-\beta-2}$. Thus (40) was established. To show the second inequality in (40) we observe that if $v<\kappa$, then it follows from (40). If $v>\kappa$ then from (39) and the lower bound of $v$, we have

$$
f(v) \leq \frac{\beta}{\beta+1} v-\frac{1}{\beta+1} \kappa+B^{-\beta} \leq M_{2} v,
$$

for some constant $C_{2}$.

In the following, we will discuss general properties of the operator $\mathcal{L}$. At first we note that it is self-adjoint on $L_{\rho}^{2}$. Its spectrum is $\operatorname{spec}(\mathcal{L})=\left\{1-\frac{m}{2} \mid m \in \mathbb{N}\right\}$; it consists of eigenvalues. The eigenfunctions of $\mathcal{L}$ are simple and derived from Hermite polynomials. For $1-\frac{m}{2}$ corresponds the eigenfunction

$$
h_{m}(y)=\sum_{n=0}^{\left[\frac{m}{2}\right]} \frac{m !}{n !(m-2 n) !}(-1)^{n} y^{m-2 n} .
$$

The polynomial $h_{m}$ satisfies $\int h_{n} h_{m} \rho d y=2^{n} n ! \delta_{n m}$. Let us introduce $k_{m}=\frac{h_{m}}{\left\|h_{m}\right\|_{L_{\rho}^{2}}^{2}}$. Since the eigenfunctions of $\mathcal{L}$ span all the space $L_{\rho}^{2}$, we expand $v$ as follows:

$$
v(y, s)=\sum_{m=0}^{2} v_{m}(s) \cdot h_{m}(y)+v_{-}(y, s)
$$

where $v_{m}$ is the projection of $v$ on $h_{m}$ and $v_{-}(y, s)=P_{-}(v)$, with $P_{-}$is the orthogonal projector on the negative subspace of $\mathcal{L}$.

Now we show that as $s \rightarrow-\infty$, either $v_{0}(s), v_{1}(s)$ or $v_{2}(s)$ is predominant with respect to the expansion (42) of $v$ in $L_{\rho}^{2}$. We have the following:

Proposition 2.7 (Classification of the behavior of $v(y, s)$ as $s \rightarrow-\infty$ ) As $s \rightarrow-\infty$, one of the following situations occurs:

(i) $\left|v_{1}(s)\right|+\left|v_{2}(., s)\right|+\left\|v_{-}(., s)\right\|_{L_{\rho}^{2}}=o\left(v_{0}(s)\right),\left\|v(., s)-C_{0} e^{s}\right\|_{L_{\rho}^{2}}=O\left(e^{\frac{3}{2} s}\right)$ for some $C_{0} \in \mathbb{R}$.

(ii) $\left|v_{0}(s)\right|+\left|v_{2}(., s)\right|+\left\|v_{-}(., s)\right\|_{L_{\rho}^{2}}=o\left(v_{1}(s)\right),\left\|v(., s)-C_{1} y e^{s / 2}\right\|_{L_{\rho}^{2}}=O\left(e^{(1-\varepsilon) s}\right)$ for some $C_{1} \in \mathbb{R} \backslash 0$ and any $\varepsilon>0$.

(iii) $\left|v_{0}(s)\right|+\left|v_{1}(s)\right|+\left\|v_{-}(., s)\right\|_{L_{\rho}^{2}}=o\left(\left\|v_{2}(., s)\right\|_{L_{\rho}^{2}}\right),\left\|v(., s)-\frac{\kappa}{4 \beta}\left(y^{2}-2\right)\right\|_{L_{\rho}^{2}}=O\left(\frac{\log (|s|)}{s^{2}}\right)$. 


\subsection{Part 3: Case (i) of Proposition 2.7: $\exists s_{0} \in \mathbb{R}$ such that $w(y, s)=$ $\varphi\left(s-s_{0}\right)$}

In this part, we prove the following:

Proposition 2.8 (The relevant case (i) of Proposition 2.7) Assume that case (i) of Proposition 2.7 holds. Then there exists $s_{0} \in \mathbb{R}$ such that for all $(y, s) \in \mathbb{R}^{2}, w(y, s)=\varphi\left(s-s_{0}\right)$, where $\varphi(s)$ is introduced in (12).

Proof: First we recall from (i) of Proposition 2.7 and the definition of $v$ that

$$
\left\|w(., s)-\left\{\kappa+C_{0} e^{s}\right\}\right\|_{L_{\rho}^{2}} \leq C e^{3 / 2 s} \text { as } s \rightarrow-\infty .
$$

Let us remark that we already have a solution $\hat{\varphi}$ of $(6)$ defined in $\mathbb{R} \times\left(-\infty, s_{1}\right]$ for some $s_{1} \in \mathbb{R}$ and which satisfies the same expansion as $w(y, s)$ when $s \rightarrow-\infty$ :

(a) if $\quad C_{0}=0$, just take $\hat{\varphi} \equiv \kappa$,

(b) if $C_{0}>0$, take $\hat{\varphi} \equiv \varphi\left(s-s_{0}\right)$ where $s_{0}=-\log \left(\frac{(\beta+1)}{\kappa} C_{0}\right)$,

(c) if $\quad C_{0}<0$, take $\hat{\varphi} \equiv \varphi_{0}\left(s-s_{0}\right)$ where $s_{0}=-\log \left(-\frac{(\beta+1)}{\kappa} C_{0}\right)$

$$
\text { and } \varphi_{0}(s)=\kappa\left(1-e^{s}\right)^{\frac{1}{\beta+1}}
$$

is a solution of (6) that quenches at $s=0$ but there exist $C>0$ such that $\varphi_{0} \geq C$ for all $s \leq-1$. If we introduce $V=w-\hat{\varphi}$, then we see from (6) that $V$ is defined for all $(y, s) \in \mathbb{R} \times\left(-\infty, s_{1}\right]$ and satisfies

$$
\frac{\partial V}{\partial s}=(\mathcal{L}+l(s)) V-F(V)
$$

where $\mathcal{L}$ is given in (39)

$$
F(V)=|\hat{\varphi}+V|^{-\beta}-\hat{\varphi}^{-\beta}+\beta \hat{\varphi}^{-(\beta+1)} V
$$

and

$$
\begin{array}{ll}
l(s)=0 & \text { if } \hat{\varphi}=\kappa, \\
l(s)=-\frac{\beta e^{s}}{(\beta+1)\left(1+e^{s}\right)} & \text { if } \hat{\varphi}=\varphi\left(s-s_{0}\right), \\
l(s)=\frac{\beta e^{s}}{(\beta+1)\left(1-e^{s}\right)} & \text { if } \hat{\varphi}=\varphi_{0}\left(s-s_{0}\right) .
\end{array}
$$

We note that $|l(s)| \leq C e^{s}$ for all $s \leq s_{1}$ and some $C>0$ and there exist $M_{i=1,2}$ such that

$$
0 \leq F(V) \leq M_{1} V^{2} \text { and } 0 \leq F(V) \leq M_{2}|V|
$$

We omit the proof of (47) since it is quite similar to the proof of (40). Let us introduce $I(s)=\|V(., s)\|_{L_{\rho}^{2}}$, multiply (45) by $V \rho$ and integrate over $\mathbb{R}$. Using the fact that 1 is the greatest eigenvalue of $\mathcal{L}$ and (47), we obtain

$$
I^{\prime}(s) \leq(1+l(s)) I(s)+C \int V^{4}(y, s) \rho d y .
$$

Now, we recall the following from [Vel93]: 
Lemma 2.9 (Regularizing effect of the operator $\mathcal{L}$ ) Assume that $\psi(y, s)$ satisfies

$$
\forall s \in[a, b], \quad \forall y \in \mathbb{R}, \quad \partial_{s} \psi \leq(\mathcal{L}+\sigma) \psi, 0 \leq \psi(y, s),
$$

for some $a \leq b$ and $\sigma \in \mathbb{R}$, where

$$
\mathcal{L} \psi=\partial_{y}^{2} \psi-\frac{1}{2} y \cdot \partial_{y} \psi+\psi=\frac{1}{\rho} \operatorname{div}\left(\rho \partial_{y} \psi\right)+\psi .
$$

Then for any $r>1$, there exists $C^{*}=C^{*}(r, \sigma)>0$ and $s^{*}(r)$, such that

$$
\forall s \in\left[a+s^{*}, b\right], \quad\left(\int_{\mathbb{R}}|\psi(y, s)|^{r} \rho(y) d y\right)^{1 / r} \leq C^{*}\left\|\psi\left(., s-s^{*}\right)\right\|_{L_{\rho}^{2}} .
$$

Proof of Lemma: See Lemma 2.3 in [HV93]

Using the lemma above, we obtain the existence of $C^{*}>0$ and $s^{*}>0$, such that $\left\|V(., s)^{2}\right\|_{L_{\rho}^{2}} \leq C^{*}\left\|V\left(., s-s^{*}\right)\right\|_{L_{\rho}^{2}}^{2}$. Then we obtain for some $s_{2} \leq s_{1}$ :

$$
\forall s \leq s_{2} \quad I^{\prime}(s) \leq \frac{5}{4} I(s)+C I\left(s-s^{*}\right)^{2}
$$

Since $I(s) \leq C e^{3 / 2 s}$ from (43), the following lemma from [NZ10] allows us to conclude.

Lemma 2.10 (Lemma 3.6 of [NZ10]) Consider I (s) a positive $C^{1}$ function such that (50) is satisfied and $0 \leq I(s) \leq C e^{3 / 2 s}$ for all $s \leq s_{2}$, for some $s_{2}$. Then, for some $s_{3} \leq s_{2}$, we have $I(s)=0$ for all $s \leq s_{3}$.

Using Lemma 2.10 , we obtain $V \equiv 0$ on $\mathbb{R} \times\left(-\infty, s_{3}\right]$. Consequently, we have

$$
\forall(y, s) \in \mathbb{R} \times\left(-\infty, s_{3}\right], \quad w(y, s)=\hat{\varphi}(s) .
$$

From the uniqueness of the Cauchy problem for equation (6) and since $w$ is defined for all $(y, s) \in \mathbb{R} \times \mathbb{R}, \hat{\varphi}$ is defined for all $(y, s) \in \mathbb{R} \times \mathbb{R}$ and (51) holds for all $(y, s) \in \mathbb{R} \times \mathbb{R}$. Therefore, case (c) in (46) cannot hold and for all $(y, s) \in \mathbb{R}^{2}, w(y, s)=\kappa$ or $w(y, s)=$ $\varphi\left(s-s_{0}\right)$. This concludes the proof of Proposition 2.8 and finishes Part 3.

\subsection{Part 4: Irrelevance of the case (iii) of Proposition 2.7}

We consider case (iii) of Proposition 2.7. In this part, we will proceed like in Step 4 in [NZ10]. The following proposition allows us to reach a contradiction.

Proposition 2.11 Assume that case (iii) of Proposition 2.7 holds. Then, there exists $\varepsilon_{0}>0$ such that

$$
\begin{aligned}
& \lim _{s \rightarrow-\infty} \sup _{|y| \leq \varepsilon_{0} \sqrt{-s}}\left|w(y, s)-G\left(\frac{y}{\sqrt{-s}}\right)\right|=0, \\
& \text { where } G(\xi)=\left((\beta+1)-\frac{(\beta+1)^{2}}{4 \beta} \xi^{2}\right)^{\frac{1}{\beta+1}}
\end{aligned}
$$


Indeed, let us first use Proposition 2.11 to find a contradiction ruling out case (iii) of Proposition 2.7, and then prove Proposition 2.11.

We fundamentally rely on the following Lemma:

Lemma 2.12 (Lemma 2.11 from [MZ98b]) Assume that $\psi(\xi, \tau)$ satisfies for all $|\xi| \leq 4 B_{1}$ and $\tau \in\left[0, \tau_{*}\right]$ :

$$
\left\{\begin{array}{l}
\partial_{\tau} \psi \leq \partial_{y y}^{2} \psi+\lambda \psi+\mu \\
\psi(\xi, 0) \leq \psi_{0}, \psi(\xi, \tau) \leq B_{2}
\end{array}\right.
$$

where $\tau_{*} \leq 1$. Then, for all $|\xi| \leq B_{1}$ and $\tau \in\left[0, \tau_{*}\right]$,

$$
\psi(\xi, \tau) \leq e^{\lambda \tau}\left(\psi_{0}+\mu+C B_{2} e^{-B_{1}^{2} / 4}\right) .
$$

Let us define $u_{s_{0}}$ by

$$
u_{s_{0}}(\xi, \tau)=(1-\tau)^{\frac{1}{\beta+1}} w(y, s) \text { where } y=\frac{\xi+\frac{\varepsilon_{0}}{2} \sqrt{-s_{0}}}{\sqrt{1-\tau}} \text { and } s=s_{0}-\log (1-\tau) .
$$

We note that $u_{s_{0}}$ is defined for all $\tau \in[0,1)$ and $\xi \in \mathbb{R}$, and that $u_{s_{0}}$ satisfies equation (1). From Lemma 2.1, we have

$$
\forall \tau \in[0,1),\left|u_{s_{0}}(., \tau)\right| \geq M(1-\tau)^{\frac{1}{\beta+1}} .
$$

The initial condition at time $\tau=0$ is $u_{s_{0}}(\xi, 0)=w\left(\xi+\frac{\varepsilon_{0}}{2} \sqrt{-s_{0}}, s_{0}\right)$. Using Proposition 2.11, we get:

$$
\sup _{|\xi|<4\left|s_{0}\right|^{1 / 4}}\left|u_{s_{0}}(\xi, 0)-G\left(\frac{\varepsilon_{0}}{2}\right)\right| \equiv g\left(s_{0}\right) \rightarrow 0 \text { as } s_{0} \rightarrow-\infty .
$$

If we define $v$, the solution of:

$$
v^{\prime}=-v^{-\beta} \text { and } v(0)=G\left(\frac{\varepsilon_{0}}{2}\right),
$$

then

$$
v(\tau)=\kappa\left(1-\frac{(\beta+1) \varepsilon_{0}^{2}}{16 \beta}-\tau\right)^{\frac{1}{\beta+1}},
$$

which quenches at time $1-\frac{(\beta+1) \varepsilon_{0}^{2}}{16 \beta}<1$. Therefore, there exists $\tau_{0}=\tau_{0}\left(\varepsilon_{0}\right)<1$, such that

$$
v\left(\tau_{0}\right)=\frac{M}{3}\left(1-\tau_{0}\right)^{\frac{1}{\beta+1}} .
$$

Now, if we consider the function

$$
\psi=\left|u_{s_{0}}-v\right|,
$$

then the following claim allows us to conclude:

Claim 2.13 For $\left|s_{0}\right|$ large enough, $\tau \in\left[0, \tau_{0}\right]$ and $|\xi| \leq 4\left|s_{0}\right|^{1 / 4}$, we have:

(i) $\partial_{\tau} \psi \leq \partial_{\xi}^{2} \psi+C\left(\varepsilon_{0}\right) \psi$,

(ii) $\psi(\xi, 0) \leq g\left(s_{0}\right)$,

(iii) $\psi(\xi, \tau) \leq C\left(\varepsilon_{0}\right)\left|s_{0}\right|^{1 / 2}$. 
Indeed, using Lemma 2.12 with $B_{1}=\left|s_{0}\right|^{1 / 4}, B_{2}=C\left(\varepsilon_{0}\right)\left|s_{0}\right|^{1 / 2}, \tau_{*}=\tau_{0}, \psi_{0}=g\left(s_{0}\right)$, $\lambda=C\left(\varepsilon_{0}\right)$ and $\mu=0$, we get for all $\tau \in\left[0, \tau_{0}\right]$,

$$
\sup _{|\xi| \leq\left|s_{0}\right|^{1 / 4}} \psi(\xi, \tau) \leq C\left(\varepsilon_{0}\right)\left(g\left(s_{0}\right)+\left|s_{0}\right|^{1 / 2} e^{-\frac{\left|s_{0}\right|^{1 / 2}}{4}}\right) \rightarrow 0 \text { as } s_{0} \rightarrow-\infty .
$$

For $\left|s_{0}\right|$ large enough and $\xi=0$, we get: $\psi\left(0, \tau_{0}\right) \leq \frac{M}{3}\left(1-\tau_{0}\right)^{1 /(\beta+1)}$ and by $(57)$

$$
\left|u_{s_{0}}\left(0, \tau_{0}\right)\right| \leq v\left(\tau_{0}\right)+\psi\left(0, \tau_{0}\right) \leq \frac{2}{3} M\left(1-\tau_{0}\right)^{1 /(\beta+1)},
$$

which is in contradiction with (54). To conclude, it remains to prove Claim 2.13.

Proof of Claim 2.13: (i) If we note by $\Psi=u_{s_{0}}-v$, then we get

$$
\begin{aligned}
\partial_{s} \Psi & =\partial_{y}^{2} \Psi-\left(u_{s_{0}}^{-\beta}-v^{-\beta}\right), \\
& =\partial_{y}^{2} \Psi+\beta \Psi \theta^{-\beta+1}, \text { for some } \theta \text { between } u_{s_{0}} \text { and } v .
\end{aligned}
$$

Using (54), (56) and (57), we have for all $\tau \in\left[0, \tau_{0}\right]$ and $\xi \in \mathbb{R}$

$$
\frac{1}{\theta} \leq \max \left(\frac{1}{u_{0}(\xi, \tau)}, \frac{1}{v(\tau)}\right) \leq C\left(\varepsilon_{0}\right), \text { for some positive } \theta .
$$

Since $\psi=|\Psi|$, using Kato's inequality, we conclude the proof of (i).

(ii) It is directly obtained from (55).

(iii) Using the definition (58) of $\psi,(53)$ and (56), we write for all $\tau \in\left[0, \tau_{0}\right]$ and $|\xi| \leq$ $4\left|s_{0}\right|^{1 / 4}$,

$$
\psi(\xi, \tau) \leq u_{s_{0}}(\xi, \tau)+v(\tau) \leq w(y, s)+\kappa, \text { where }(y, s) \text { are defined in (53). }
$$

Since we have from Lemma 2.1, (37) and (53) for $\left|s_{0}\right|$ large enough

$$
|w(y, s)| \leq|w(0, s)|+M_{0}|y| \leq C(1+|y|)
$$

and

$$
|y| \leq \frac{\left|\xi+\frac{\varepsilon_{0}}{2} \sqrt{-s_{0}}\right|}{\sqrt{1-\tau}} \leq \frac{4\left|s_{0}\right|^{1 / 4}+\frac{\varepsilon_{0}}{2} \sqrt{-s_{0}}}{\sqrt{1-\tau_{0}}} \leq C\left(\varepsilon_{0}\right)\left|s_{0}\right|^{1 / 2},
$$

the bound on $\psi$ follows from (59). This concludes the proof of claim 2.13.

It remains to prove Propossition 2.11 to conclude Part 4 .

Proof of Proposition 2.11: Consider some arbitrary $\varepsilon_{0} \in\left(0, R^{*}\right)$, where

$$
R^{*}=\sqrt{\frac{4 \beta}{\beta+1}} .
$$

The parameter $\varepsilon_{0}$ will be fixed later in the proof small enough. If we note

$$
f(y, s)=G\left(\frac{y}{\sqrt{-s}}\right)
$$


and

$$
F(y, s)=f(y, s)-\frac{\kappa}{2 \beta s}
$$

then $f$ satisfies

$$
-\frac{y}{2} \cdot \partial_{y} f+\frac{1}{(\beta+1)} f-|f|^{-\beta}=0 .
$$

and we see from (iii) of Proposition 2.7 that

$$
\left\|(F(., s)-w(., s))\left(1-\chi_{\varepsilon_{0}}\right)\right\|_{L_{\rho}^{2}}=O\left(\frac{\log |s|}{s^{2}}\right) \text { as } s \rightarrow-\infty,
$$

where

$$
\chi_{\varepsilon_{0}}(y, s)=1 \text { if } \frac{|y|}{\sqrt{|s|}} \geq 3 \varepsilon_{0} \text { and zero otherwise. }
$$

The formal idea of this proof is that $F$ solves in an approximate way the same equation as $w$ for $s \rightarrow-\infty$. By (63), $w$ and $F$ are very close in the region $|y| \sim 1$. Our task is to prove that they remain close in the larger region $|y| \leq \varepsilon_{0} \sqrt{-s}$, for some $\varepsilon_{0}$ chosen later. Let us consider a cut-off function

$$
\gamma(y, s)=\gamma_{0}\left(\frac{y}{\sqrt{-s}}\right)
$$

where $\gamma_{0} \in \mathcal{C}^{\infty}(\mathbb{R})$ is such that $\gamma_{0}(\xi)=1$ if $|\xi| \leq 3 \varepsilon_{0}$ and $\gamma_{0}(\xi)=0$ if $|\xi| \geq 4 \varepsilon_{0}$. We introduce

$$
\nu=w-F \text { and } Z=\gamma|\nu| .
$$

Our proof is the same as Velázquez [Vel92] and Nouaili and Zaag [NZ10]. As in [NZ10], we need to multiply by the cut-off, since our profile $F(y, s)$ defined by $(62)$ is singular on the parabola $y=R^{*} \sqrt{-s}$. The cut-off function will generate an extra term, difficult to handle. Let us present the major steps of the proof in the following. The proof of the presented Lemmas will be given at the end of this step.

Lemma 2.14 (Estimates in modified $L_{\rho}^{2}$ spaces) There exists $\varepsilon_{0}>0$ such that the function $Z$ satisfies for all $s \leq s_{*}$ and $y \in \mathbb{R}$ :

$\partial_{s} Z-\partial_{y}^{2} Z+\frac{1}{2} y \cdot \partial_{y} Z-(1+\sigma) Z \leq C\left(Z^{2}+\frac{\left(y^{2}+1\right)}{s^{2}}+(1+\sqrt{-s}) \chi_{\varepsilon_{0}}\right)-2 \operatorname{div}\left(|\nu| \cdot \partial_{y} \gamma\right)$,

where $s_{*} \in \mathbb{R}, \sigma=1 / 100$ and $\chi_{\varepsilon_{0}}$ is defined in (64). Moreover,

$$
N_{2 \varepsilon_{0} \sqrt{|s|}}^{2}(Z(s))=o(1) \text { as } s \rightarrow-\infty,
$$

where the norm $N_{r}^{q}(\psi)$ is defined, for all $r>0$ and $1 \leq q<\infty$, by

$$
N_{r}^{q}(\psi)=\sup _{|\xi| \leq r}\left(\int|\psi(y)|^{q} \exp \left(-\frac{(y-\xi)^{2}}{4}\right) d y\right)^{1 / q} .
$$


Using the regularizing effect of the operator $\mathcal{L}$, we derive the following pointwise estimate, which allows us to conclude the proof of Proposition 2.11:

Lemma 2.15 (An upper bound for $Z(y, s)$ in $\left\{|y| \leq \varepsilon_{0} \sqrt{-s}\right\}$ ) We have:

$$
\sup _{|y| \leq \varepsilon_{0} \sqrt{-s}} Z(y, s)=o(1) \text { as } s \rightarrow-\infty .
$$

Thus, Proposition 2.11 follows from Lemma 2.15 by (66), (62) and (61). It remains to prove Lemmas 2.14 and 2.15 .

Proof of Lemma 2.14: The proof of (67) is straightforward and a bit technical. We leave it to Appendix B. Let us then prove (68). We take $s_{0}<s^{*}$ and $s_{0} \leq s<s^{*}$ such that $e^{\frac{s-s_{0}}{2}} \leq \sqrt{-s}$. We use the variation of constant formula in (67) to write

$$
\begin{aligned}
Z(y, s) \leq & S_{\sigma}\left(s-s_{0}\right) Z\left(., s_{0}\right) \\
& +\int_{s_{0}}^{s} S_{\sigma}(s-\tau)\left(C\left\{Z^{2}+\frac{\left(y^{2}+1\right)}{\tau^{2}}+(1+\sqrt{-\tau}) \chi_{\varepsilon_{0}}\right\}-2 \operatorname{div}\left(|\nu| \partial_{y} \gamma\right)\right) d \tau
\end{aligned}
$$

where $S_{\sigma}$ is the semigroup associated to the operator $\mathcal{L}_{\sigma} \phi=\partial_{y}^{2} \phi-\frac{1}{2} y \partial_{y} \phi+(1+\sigma) \phi$, defined on $L_{\rho}^{2}(\mathbb{R})$. The kernel of the semigroup $S_{\sigma}(\tau)$ is

$$
S_{\sigma}(\tau, y, z)=\frac{e^{(1+\sigma) \tau}}{\left(4 \pi\left(1-e^{-\tau}\right)\right)^{1 / 2}} \exp \left[-\frac{\left|y e^{-\tau / 2}-z\right|^{2}}{4\left(1-e^{-\tau}\right)}\right] .
$$

Setting

$$
r \equiv r\left(s, s_{0}\right)=2 \varepsilon_{0} e^{\frac{s-s_{0}}{2}}=R_{1} e^{\frac{s-s_{0}}{2}}
$$

and taking the $N_{r}^{2}$-norm we obtain

$$
\begin{array}{r}
N_{r}^{2}(Z(., s)) \leq N_{r}^{2}\left(S_{\sigma}\left(s-s_{0}\right) Z\left(., s_{0}\right)\right)+C \int_{s_{0}}^{s} N_{r}^{2}\left(S_{\sigma}(s-\tau) Z(., \tau)^{2}\right) d \tau \\
+C \int_{s_{0}}^{s} N_{r}^{2}\left(S_{\sigma}(s-\tau)\left(\frac{\left(y^{2}+1\right)}{\tau^{2}}\right)\right) d \tau \\
+C \int_{s_{0}}^{s} N_{r}^{2}\left(S_{\sigma}(s-\tau)(1+\sqrt{-\tau}) \chi_{\varepsilon_{0}}(y, \tau)\right) d \tau+C \int_{s_{0}}^{s} N_{r}^{2}\left(S_{\sigma}(s-\tau)\left(\operatorname{div}\left(|\nu| \partial_{y} \gamma\right)\right) d \tau\right. \\
\equiv J_{1}+J_{2}+J_{3}+J_{4}+J_{5} .
\end{array}
$$

In comparison with [Vel92], we have a new term $J_{5}$ coming from the cut-off terms. Therefore, we just recall in the following claim the estimates on $J_{1} \ldots J_{4}$ from [Vel92], and treat $J_{2}$ and $J_{5}$, which are new ingredients in our proof: 
Claim 2.16 We obtain as $s \rightarrow-\infty$

$$
\begin{aligned}
&\left|J_{1}\right| \leq C e^{(1+\sigma)\left(s-s_{0}\right)} \frac{\log \left|s_{0}\right|}{\left|s_{0}^{2}\right|}, \\
&\left|J_{2}\right| \leq C \int_{s_{0}}^{s_{0}+\left(\left(s-R_{0}\right)-s_{0}\right)+} \frac{e^{(1+\sigma)\left(s-\tau-R_{0}\right)}}{\left(1-e^{s-\tau-R_{0}}\right)^{1 / 20}}\left(L_{r}^{2}\left(Z(., \tau)^{2}\right)\right) d \tau+C \frac{e^{\left(s-s_{0}\right)(1+\sigma)}}{s_{0}^{2}}, \\
& \text { with } R_{0}=4 \varepsilon_{0}, \\
&\left|J_{3}\right| \leq C \frac{e^{\left(s-s_{0}\right)(1+\sigma)}}{s_{0}^{2}}\left(1+\left(s-s_{0}\right)\right) \sqrt{-s_{0}}, \\
&\left|J_{4}\right| \leq C e^{\left(s-s_{0}\right)(1+\sigma)} e^{\alpha s}, \text { where } \alpha>0, \\
&\left|J_{5}\right| \leq C e^{\left(s-s_{0}\right)(1+\sigma)} e^{\beta s}, \text { where } \beta>0 .
\end{aligned}
$$

Proof: See page 1578 in [Vel92] for $J_{1}, J_{3}$ and $J_{4}$.

To obtain the bound on $J_{2}$, we need the following inequality, which will be proved in Appendix B

$$
\partial_{s} Z-\partial_{y}^{2} Z+\frac{1}{2} y \cdot Z \leq C\left(Z+\frac{\left(y^{2}+1\right)}{s^{2}}+(1+\sqrt{-s}) \chi_{\varepsilon_{0}}\right)-2 \operatorname{div}\left(|\nu| \cdot \partial_{y} \gamma\right),
$$

then, we proceed exactly as in page 1579-1580 in [Vel92] and we obtain the wanted estimation on $J_{2}$.

Now, we treat $J_{5}$. We have from (70):

$$
\begin{aligned}
& S_{\sigma}(s-\tau)\left(-\operatorname{div}\left(\mid \nu \partial_{y} \gamma\right)\right) \\
& =-\frac{C e^{(s-\tau)(1+\sigma)}}{\left(1-e^{s-\tau}\right)^{1 / 2}} \int_{\mathbb{R}} \exp \left(-\frac{\left(y e^{-(s-\tau) / 2}-\lambda\right)^{2}}{4\left(1-e^{-(s-\tau)}\right)}\right) \operatorname{div}\left(|\nu| \partial_{y} \gamma\right) d \lambda, \\
& =\frac{C e^{(s-\tau)(1+\sigma)}}{\left(1-e^{s-\tau}\right)^{1 / 2}} \int_{\mathbb{R}}-\frac{\left(y e^{-(s-\tau) / 2}-\lambda\right)}{2\left(1-e^{-(s-\tau)}\right)} \exp \left(-\frac{\left(y e^{-(s-\tau) / 2}-\lambda\right)^{2}}{4\left(1-e^{-(s-\tau)}\right)}\right)|\nu| \partial_{y} \gamma d \lambda .
\end{aligned}
$$

We have from Lemma 2.1 and (37)

$$
|w(y, s)| \leq|w(0, s)|+M_{0}|y| \leq C(1+|y|) .
$$

Since $F$ is bounded for $\frac{|y|}{\sqrt{-\tau}} \leq R^{*} / 2$, where $R^{*}$ is defined by (60) and $\operatorname{supp}\left(\partial_{y} \gamma\right) \subset$ $\left(-4 \varepsilon_{0} \sqrt{-\tau},-3 \varepsilon_{0} \sqrt{-\tau}\right) \cup\left(3 \varepsilon_{0} \sqrt{-\tau}, 4 \varepsilon_{0} \sqrt{-\tau}\right)$, we have

$$
\begin{aligned}
\| \nu\left|\partial_{y} \gamma\right| & \leq C|\nu| \mathbb{I}_{\left\{3 \varepsilon_{0} \leq \frac{|y|}{\sqrt{-\tau}} \leq 4 \varepsilon_{0}\right\}}, \\
& \leq C(1+\sqrt{-\tau})\left(\mathbb{I}_{3 \varepsilon_{0} \leq \frac{|y|}{\sqrt{-\tau}} \leq 4 \varepsilon_{0}}\right) \leq C(1+\sqrt{-\tau}) \chi_{\varepsilon_{0}} .
\end{aligned}
$$

Using Cauchy-Schwartz inequality, we obtain:

$$
\left|S_{\sigma}(s-\tau)\left(-\operatorname{div}\left(|\nu| \partial_{y} \gamma\right)\right)\right| \leq \frac{C e^{(s-\tau)(1+\sigma)}(1+\sqrt{-\tau})}{\left(1-e^{s-\tau}\right)^{3 / 2}} \mathcal{I}_{1} \mathcal{I}_{2},
$$


where,

$$
\begin{aligned}
& \mathcal{I}_{1}=\left(\int_{\mathbb{R}}\left(y e^{-(s-\tau) / 2}-\lambda\right)^{2} \exp \left(-\frac{\left(y e^{-(s-\tau) / 2}-\lambda\right)^{2}}{4\left(1-e^{-(s-\tau)}\right)}\right) d \lambda\right)^{1 / 2}, \\
& \mathcal{I}_{2}=\left(\int_{\mathbb{R}} \exp \left(-\frac{\left(y e^{-(s-\tau) / 2}-\lambda\right)^{2}}{4\left(1-e^{-(s-\tau)}\right)}\right) \chi_{\varepsilon_{0}} d \lambda\right)^{1 / 2} .
\end{aligned}
$$

Doing a change of variables, we obtain $\mathcal{I}_{1}=C\left(1-e^{-(s-\tau)}\right)^{3 / 4}$. Furthermore, we have:

$$
\mathcal{I}_{2}^{2} \leq \mathcal{I}_{3}\left(\int_{\mathbb{R}} \chi_{\varepsilon_{0}} e^{-\frac{\lambda^{2}}{4}} d \lambda\right)^{1 / 2}
$$

where,

$$
\mathcal{I}_{3}=\left(\int \exp \left(-\frac{\left(y e^{-(s-\tau) / 2}-\lambda\right)^{2}}{2\left(1-e^{-(s-\tau)}\right)}+\frac{\lambda^{2}}{4}\right) d \lambda\right)^{1 / 2} .
$$

We introduce $\theta=y e^{-(s-\tau) / 2}$, by completing squares, we readily check that:

$$
\frac{\lambda^{2}}{4}-\frac{(\theta-\lambda)^{2}}{2\left(1-e^{-(s-\tau)}\right)}=-\frac{\left(1+e^{-(s-\tau)}\right)}{4\left(1-e^{-(s-\tau)}\right)}\left(\lambda-\frac{2 \theta}{\left(1+e^{-(s-\tau)}\right)}\right)^{2}+\frac{\theta^{2}}{2\left(1+e^{-(s-\tau)}\right)},
$$

then we obtain:

$$
\mathcal{I}_{3}^{2}=C\left(\frac{\left(1-e^{-(s-\tau)}\right)}{\left(1+e^{-(s-\tau)}\right)}\right)^{1 / 2} \exp \left(\frac{\theta^{2}}{2\left(1-e^{-(s-\tau)}\right)}\right)
$$

Therefore,

$$
\begin{aligned}
\left|N_{r}^{2}\left(S_{\sigma}(s-\tau) \operatorname{div}\left(|\nu| \partial_{y} \gamma\right)\right)\right| & \leq C \frac{e^{(s-\tau)(1+\sigma)}(1+\sqrt{-\tau})}{\left(1+e^{-(s-\tau)}\right)^{1 / 8}\left(1-e^{-(s-\tau)}\right)^{5 / 8}}\left\|\chi_{\varepsilon_{0}}\right\|_{L_{\rho}^{2}}^{1 / 2} \mathcal{I}_{4}, \\
\text { where } \mathcal{I}_{4} & =N_{r}^{2}\left(\exp \left(\frac{y^{2} e^{-(s-\tau)}}{8\left(\left(1-e^{-(s-\tau)}\right)\right)}\right)\right) .
\end{aligned}
$$

Let us compute $\mathcal{I}_{4}$. Using the fact that

$$
\begin{aligned}
& -\frac{(y-\mu)^{2}}{4}+\frac{y^{2} e^{-(s-\tau)}}{4\left(1-e^{-(s-\tau)}\right)}= \\
& \frac{1}{4}\left(-\left(y\left(1+e^{-(s-\tau)}\right)^{-1 / 2}-\mu\left(1+e^{-(s-\tau)}\right)^{1 / 2}\right)^{2}+\mu^{2} e^{-(s-\tau)}\right),
\end{aligned}
$$

and doing a change of variables, we obtain:

$$
\begin{aligned}
& \int_{\mathbb{R}} \exp \left(-\frac{(y-\mu)^{2}}{4}+\frac{y^{2} e^{-(s-\tau)}}{4\left(1-e^{(s-\tau)}\right)}\right) d y \\
& \leq C \exp \left(\frac{\mu^{2} e^{-(s-\tau)}}{4}\right) \int_{\mathbb{R}} \exp \left(-\frac{1}{4}\left(y\left(1+e^{-(s-\tau)}\right)^{-1 / 2}-\mu\left(1+e^{-(s-\tau)}\right)^{1 / 2}\right)^{2}\right) d y .
\end{aligned}
$$


Hence $\mathcal{I}_{4} \leq C\left(1+e^{-(s-\tau)}\right)^{1 / 8}$ and

$$
\left.N_{r}^{2}\left(S_{\sigma}(s-\tau)\right) \operatorname{div}\left(|\nu| \partial_{y} \gamma\right)\right) \leq C \frac{e^{(s-\tau)(1+\sigma)}(1+\sqrt{-\tau})}{\left(1-e^{-(s-\tau)}\right)^{5 / 8}}\left(\int_{|\lambda| \geq R_{1} \sqrt{-\tau}} e^{-\frac{\lambda^{2}}{4}} d \lambda\right) .
$$

This gives

$$
\left|J_{5}\right|=\int_{s_{0}}^{s} N_{r}^{2}\left(S_{\sigma}(s-\tau)\left(\operatorname{div}\left(|\nu| \partial_{y} \gamma\right)\right) d \tau \leq C(\eta) e^{\left(s-s_{0}\right)(1+\sigma)} e^{\alpha s_{0}},\right.
$$

where $\alpha>0$. This concludes the proof of the claim 2.16.

This concludes the proof of Claim 2.16.

Summing up $J_{i=1 . .5}$, from claim 2.16 we obtain

$$
\begin{aligned}
& N_{r}^{2}(Z(., s)) \leq \\
& e^{\left(s-s_{0}\right)(1+\sigma)} C \frac{\log \left|s_{0}\right|}{s_{0}^{2}}+C \int_{s_{0}}^{s_{0}+\left(\left(s-R_{0}\right)-s_{0}\right)+} \frac{e^{\left(s-\tau-R_{0}\right)(1+\sigma)}}{\left(1-e^{s-\tau-R_{0}}\right)^{1 / 20}}\left(N_{r}^{2}(Z(., \tau))\right)^{2} d \tau .
\end{aligned}
$$

Now, we recall the following from [Vel92]:

Lemma 2.17 Let $\varepsilon, C, R, \sigma$ and $\alpha$ be positives constants, $0<\alpha<1$ and assume that $H(s)$ is a family of continuous functions satisfying:

$$
H(s) \leq \varepsilon e^{s(1+\sigma)}+C \int_{0}^{(s-R)_{+}} \frac{e^{(s-\tau)(1+\sigma)} H(\tau)^{2}}{\left(1-e^{(s-\tau-R)}\right)^{\alpha}} d \tau \text { for } s>0 .
$$

Then there exists $\xi=\xi(R, C, \alpha)$ such that for any $\varepsilon \in\left(0, \varepsilon_{1}\right)$ and any $s$ for which $\varepsilon e^{s(1+\sigma)} \leq$ $\xi$, we have

$$
H(s) \leq 2 \varepsilon e^{s(1+\sigma)}
$$

Proof: See the proof of Lemma 2.2 from [Vel92]. Note that the proof of [Vel92] is done in the case $\sigma=0$, but it can be adapted to some $\sigma>0$ with no difficulty.

We conclude that $N_{r\left(\tau, s_{0}\right)}^{2}(Z(., s)) \leq C e^{\left(s-s_{0}\right)(1+\sigma) \frac{\log \left|s_{0}\right|}{s_{0}^{2}}}$ as $s \rightarrow-\infty$. If we fix $s=-e^{\left(s-s_{0}\right)}$, then we obtain $s \sim s_{0}, \log |s| \sim \log \left|s_{0}\right|$ and $N_{R_{1} \sqrt{-s}}^{2}(Z(., s)) \leq C s^{1+\sigma} \frac{\log \left|s_{0}\right|}{s_{0}^{2}} \leq C \frac{\log |s|}{s^{1-\sigma}} \rightarrow 0$ as $s \rightarrow-\infty$. Since $\sigma=\frac{1}{100}$, we get $N_{R_{1} \sqrt{-s}}^{2}(Z(., s))=o(1)$, as $s \rightarrow-\infty$.

This concludes the proof of Lemma 2.14

Now we give the proof of Lemma 2.15.

Proof of Lemma 2.15.

We aim at bounding $Z(y, s)$ for $|y| \leq R_{2} \sqrt{-s}$ in terms of $N_{R_{1} \sqrt{-s^{\prime}}}\left(Z\left(s^{\prime}\right)\right)$, where $R_{2}=\varepsilon_{0}$ and $R_{1}=2 \varepsilon_{0}$, for some $s^{\prime}<s$. Starting from equation (72), we do as in [Vel92]:

$$
\begin{aligned}
Z(., s) \leq & \left\{e^{C R_{0}} S\left(R_{0}\right) Z\left(., s-R_{0}\right)\right\} \\
& +\left\{C \int_{s-R_{0}}^{s} e^{C(s-\tau)} S(s-\tau)\left(\frac{\left(y^{2}+1\right)}{\tau^{2}}+(1+\sqrt{-\tau}) \chi_{\varepsilon_{0}}\right) d \tau\right\} \\
& -\left\{2 \int_{s-R_{0}}^{s} e^{C(s-\tau)} S(s-\tau)\left(\operatorname{div}\left(|\nu| \partial_{y} \gamma\right)\right) d \tau\right\} \\
= & \mathcal{M}_{1}+\mathcal{M}_{2}+\mathcal{M}_{3}, \text { where } R_{0}=4 \varepsilon_{0},
\end{aligned}
$$


where $S$ is the semigroup associated to the operator $\mathcal{L}$ defined in (48). The terms $\mathcal{M}_{1}$ and $\mathcal{M}_{2}$ are estimated in the following:

Claim 2.18 (Velázquez) There exists $s_{0}$, such that for all $s \leq s_{0}$

$$
\begin{aligned}
& \sup _{|y| \leq R_{2} \sqrt{-s}}\left|\mathcal{M}_{1}\right|=\sup _{|y| \leq R_{2} \sqrt{-s}}\left|e^{C R_{0}} S\left(R_{0}\right) Z\left(., s-R_{0}\right)\right|=o(1) \text { as } s \rightarrow-\infty \\
& \sup _{|y| \leq R_{2} \sqrt{-s}}\left|\mathcal{M}_{2}\right|=\sup _{|y| \leq R_{2} \sqrt{-s}} \int_{s-R_{0}}^{s}\left(\frac{|y|^{2}+1}{s^{2}}+(1+\sqrt{-\tau}) \chi_{\varepsilon_{0}}\right) \leq \frac{C}{|s|}
\end{aligned}
$$

Proof: See page 1581 from [Vel92] and Lemma 6.5 in [HV93] in a similar case.

It remains to estimate $\mathcal{M}_{3}$. Proceeding as in page (73) and using the fact that $\nu(y, s) \leq$ $C(1+\sqrt{-s}$ ) for all $|y| \leq C \sqrt{-s}$ (obtained from (i) of Lemma 2.1) we write

$$
\begin{aligned}
& \left|S(s-\tau)\left(-\operatorname{div}\left(|\nu| \partial_{y} \gamma\right)\right)\right| \\
& =\left|\frac{C e^{s-\tau}}{\left(1-e^{s-\tau}\right)^{1 / 2}} \int_{\mathbb{R}} \exp \left(-\frac{\left(y e^{-(s-\tau) / 2}-\lambda\right)^{2}}{4\left(1-e^{-(s-\tau)}\right)}\right) \operatorname{div}\left(|\nu| \partial_{y} \gamma\right) d \lambda\right|, \\
& =\mid \frac{C e^{s-\tau}}{\left(1-e^{s-\tau}\right)^{1 / 2}} \int_{\mathbb{R}}-\frac{\left(y e^{-(s-\tau) / 2}-\lambda\right)}{2\left(1-e^{-(s-\tau)}\right)} \exp \left(-\frac{\left(y e^{-(s-\tau) / 2}-\lambda\right)^{2}}{4\left(1-e^{-(s-\tau)}\right)}\right)\left(|\nu| \partial_{y} \gamma d \lambda \mid,\right. \\
& \leq \frac{C e^{s-\tau}}{\left(1-e^{s-\tau}\right)^{3 / 2}} \int_{\mathbb{R}}\left|y e^{-(s-\tau) / 2}-\lambda\right| \exp \left(-\frac{\left(y e^{-(s-\tau) / 2}-\lambda\right)^{2}}{4\left(1-e^{-(s-\tau)}\right)}\right) \chi_{\varepsilon_{0}} d \lambda, \\
& \leq \frac{C e^{s-\tau} \sqrt{-\tau}(1+\sqrt{-\tau})}{\left(1-e^{s-\tau}\right)^{3 / 2}} \int_{\mathbb{R}} \exp \left(-\frac{\left(y e^{-(s-\tau) / 2}-\lambda\right)^{2}}{4\left(1-e^{-(s-\tau)}\right)}\right) \chi_{\varepsilon_{0}} d \lambda .
\end{aligned}
$$

We make the change of variables $z=\left(1-e^{-(s-\tau)}\right)^{-1 / 2}\left(\lambda-e^{-(\tau-s) / 2} y\right)$ and we obtain

$$
\int_{\mathbb{R}} \exp \left(-\frac{\left(y e^{-(s-\tau) / 2}-\lambda\right)^{2}}{4\left(1-e^{-(s-\tau)}\right)}\right) \chi_{\varepsilon_{0}} d \lambda \leq\left(1-e^{s-\tau}\right)^{1 / 2} \int_{\Sigma} e^{-z^{2} / 4} d z,
$$

where,

$$
\Sigma=\left\{z \in \mathbb{R}:\left|z+e^{-(\tau-s) / 2}\left(1-e^{s-\tau}\right)^{-1 / 2} y\right| \geq 3 \varepsilon_{0}\left(1-e^{s-\tau}\right)^{-1 / 2} \sqrt{-\tau}\right\} .
$$

Since $\left|y e^{-(\tau-s) / 2}\right| \leq \varepsilon_{0} \sqrt{-s}$, we readily see that $\Sigma \subset\left\{z \in \mathbb{R}:|z| \geq \varepsilon_{0} \sqrt{-s}\right\}$. Then we conclude that

$$
\left|S(s-\tau)\left(-\operatorname{div}\left(|\nu| \partial_{y} \gamma\right)\right)\right| \leq \frac{C e^{s-\tau}}{\left(1-e^{s-\tau}\right)} e^{\beta s}, \text { where } \beta>0,
$$

and we obtain

$$
\sup _{|y| \leq R_{2} \sqrt{-s}}\left|\mathcal{M}_{3}\right|=o\left(\frac{1}{|s|}\right) \text { as } s \rightarrow-\infty \text {. }
$$

Putting together $\mathcal{M}_{i=1 . .3}$, the proof of lemma 2.15 is complete. This concludes also the proof of Proposition 2.11 and rules out case (iii) of Proposition 2.7. 


\subsection{Part 5: Irrelevance of the case (ii) of Proposition 2.7}

To conclude the proof of Theorem 1, we consider case (ii) of Proposition 2.7. We claim that the following proposition allows us to reach a contradiction in this case.

\section{Proposition 2.19}

$$
\lim _{s \rightarrow-\infty} \sup _{|y| \leq \varepsilon_{0} e^{-s / 2}}\left|w(y, s)-G\left(y e^{-s / 2}\right)\right|=0 \text {, where } G(\xi)=\kappa\left(1-C_{1} \kappa^{\beta} \xi\right)^{\frac{1}{\beta+1}} .
$$

As in the previous part, first, we will find a contradiction ruling out case (ii) of Proposition 2.7 and then prove Proposition 2.19.

Let us define $u_{s_{0}}$ by

$$
u_{s_{0}}(\xi, \tau)=(1-\tau)^{\frac{1}{\beta+1}} w(y, s) \text { where } y=\frac{\xi+\frac{\varepsilon_{0}}{2} e^{-s_{0} / 2}}{\sqrt{1-\tau}} \text { and } s=s_{0}-\log (1-\tau) .
$$

We note that $u_{s_{0}}$ is defined for all $\tau \in[0,1)$ and $\xi \in \mathbb{R}$, and that $u_{s_{0}}$ satisfies equation (1). From Lemma 2.1, we have

$$
\forall \tau \in[0,1),\left|u_{s_{0}}(., \tau)\right| \geq M(1-\tau)^{\frac{1}{\beta+1}} .
$$

The initial condition at time $\tau=0$ is $u_{s_{0}}(\xi, 0)=w\left(\xi+\frac{\varepsilon_{0}}{2} e^{-s_{0} / 2}, s_{0}\right)$. Using Proposition 2.11, we get:

$$
\sup _{|\xi|<4 e^{-s_{0} / 4}}\left|u_{s_{0}}(\xi, 0)-G\left(\frac{\varepsilon_{0}}{2}\right)\right| \equiv g\left(s_{0}\right) \rightarrow 0 \text { as } s_{0} \rightarrow-\infty .
$$

If we define $v$, the solution of:

$$
v^{\prime}=-v^{-\beta} \text { and } v(0)=G\left(\frac{\varepsilon_{0}}{2}\right),
$$

then

$$
v(\tau)=\kappa\left(1-C_{1} \kappa^{\beta} \frac{\varepsilon_{0}}{2}-\tau\right)^{\frac{1}{\beta+1}},
$$

which quenches at time $1-C_{1} \kappa^{\beta} \frac{\varepsilon_{0}}{2}<1$. Therefore, there exists $\tau_{0}=\tau_{0}\left(\varepsilon_{0}\right)<1$, such that

$$
v\left(\tau_{0}\right)=\frac{M}{3}\left(1-\tau_{0}\right)^{\frac{1}{\beta+1}} .
$$

Now, if we consider the function

$$
\psi=\left|u_{s_{0}}-v\right|,
$$

then the following claim allows us to conclude (we omit the proof since it is the same as the proof of Lemma 2.13):

Claim 2.20 For $\left|s_{0}\right|$ large enough and for all $\tau \in\left[0, \tau_{0}\right]$ and $|\xi| \leq 4 e^{-s_{0} / 4}$, we have:

(i) $\partial_{\tau} \psi \leq \partial_{\xi}^{2} \psi+C\left(\varepsilon_{0}\right) \psi$,

(ii) $\psi(\xi, 0) \leq g\left(s_{0}\right)$,

(iii) $\psi(\xi, \tau) \leq C\left(\varepsilon_{0}\right) e^{-s_{0} / 2}$. 
Indeed, using Lemma 2.12 with $B_{1}=e^{-s_{0} / 4}, B_{2}=C\left(\varepsilon_{0}\right) e^{-s_{0} / 2}, \tau_{*}=\tau_{0}, \psi_{0}=g\left(s_{0}\right)$, $\lambda=C\left(\varepsilon_{0}\right)$ and $\mu=0$, we get for all $\tau \in\left[0, \tau_{0}\right]$,

$$
\sup _{|\xi| \leq e^{-s_{0} / 4}} \psi(\xi, \tau) \leq C\left(\varepsilon_{0}\right)\left(g\left(s_{0}\right)+e^{-s_{0} / 2} e^{-\frac{e^{-s_{0} / 2}}{4}}\right) \rightarrow 0 \text { as } s_{0} \rightarrow-\infty .
$$

For $\left|s_{0}\right|$ large enough and $\xi=0$, we get: $\psi\left(0, \tau_{0}\right) \leq \frac{M}{3}\left(1-\tau_{0}\right)^{1 /(\beta+1)}$ and by $(57)$

$$
\left|u_{s_{0}}\left(0, \tau_{0}\right)\right| \leq v\left(\tau_{0}\right)+\psi\left(0, \tau_{0}\right) \leq \frac{2}{3} M\left(1-\tau_{0}\right)^{1 /(\beta+1)},
$$

which is in contradiction with (54).

Proof of Proposition 2.19: The proof is very similar to that of Proposition 2.11. If we note $f(y, s)=G\left(y e^{s / 2}\right)$, then $f$ satisfies

$$
-\partial_{s} f-\frac{1}{2} y \cdot \partial_{y} f+\frac{f}{\beta+1}-f^{-\beta}=0 .
$$

Consider an arbitrary $\varepsilon_{0} \in\left(0, \frac{R^{*}}{10}\right)$, where $R^{*}=\frac{\kappa^{p}}{C_{1}} \cdot \varepsilon_{0}$ will be fixed small enough later. Let us consider a cut-off function $\gamma(y, s)=\gamma_{0}\left(y e^{s / 2}\right)$, where $\gamma_{0} \in \mathcal{C}^{\infty}(\mathbb{R})$ such that $\gamma_{0}(\xi)=1$ if $|\xi| \leq 3 \varepsilon_{0}$ and $\gamma_{0}(\xi)=0$ if $|\xi| \geq 4 \varepsilon_{0}$. We note $\nu=w-f$ and $Z=\gamma|\nu|$. From (ii) of Proposition 2.7, we have

$$
\|Z\|_{L_{\rho}^{2}} \leq C e^{s(1-\varepsilon)} \text { as } s \rightarrow-\infty \text {, for some } \varepsilon>0 .
$$

As in the previous part, we divide our proof in two parts given in the following lemmas.

Lemma 2.21 (Estimates in the modified $L_{\rho}^{2}$ spaces) There exists $\varepsilon_{0}>0$ such that the function $Z$ satisfies for all $s \leq s_{*}$ and $y \in \mathbb{R}$,

$$
\partial_{s} Z-\partial_{y}^{2} Z+\frac{1}{2} y \cdot \partial_{y} Z-(1+\sigma) Z \leq C\left(Z^{2}+e^{s}+\left(1+e^{-s / 2}\right) \chi_{\varepsilon_{0}}\right)-2 \operatorname{div}\left(|\nu| \partial_{y} \gamma\right),
$$

where $s_{*} \in \mathbb{R}, \sigma=\frac{1}{100}$ and

$$
\chi_{\varepsilon_{0}}(y, s)=1 \text { if }|y| e^{s / 2} \geq 3 \varepsilon_{0} \text { and zero otherwise. }
$$

Moreover, we have

$$
N_{2 \varepsilon_{0} e^{-s / 2}}^{2}(Z(s))=o(1) \text { as } s \rightarrow-\infty \text {. }
$$

As in Part 4, the following lemma allows us to conclude the proof of Proposition 2.19:

Lemma 2.22 (An upper bound for $Z(y, s)$ in $|y| \leq \varepsilon_{0} e^{-s / 2}$ ) We have:

$$
\sup _{|y| \leq \varepsilon_{0} e^{-s / 2}} Z(y, s)=o(1) \text { as } s \rightarrow-\infty .
$$


Remains to prove Lemmas 2.21 and 2.22 to conclude the proof of Proposition 2.19. Here, we only sketch the proof of Lemma 2.21, since it is completely similar to Part 4 . We don't give the proof of Lemma 2.22. We refer the reader to Part 4 and Proposition 2.4 from Velázquez [Vel92] for similar situations.

Proof of Lemma 2.21: As in the previous step, we leave the proof of (84) to Appendix B. Let us now apply the variation of constants formula and take the norm $N_{r\left(s, s_{0}\right)}^{2}$, where $r\left(s, s_{0}\right)$ is as in (71). Assume that $s_{0}<2 s_{*}$, then for all $s_{0} \leq s \leq \frac{s_{0}}{2}$, we have

$$
\begin{aligned}
N_{r}^{2}(Z(., s)) \leq & N_{r}^{2}\left(S_{\sigma}\left(s-s_{0}\right) Z\left(., s_{0}\right)\right)+C \int_{s_{0}}^{s} N_{r}^{2}\left(S_{\sigma}(s-\tau)\left(Z(., \tau)^{2}\right)\right) d \tau \\
& +C \int_{s_{0}}^{s} N_{r}^{2}\left(S(s-\tau)\left(e^{\tau}\right)\right) d \tau \\
& +C \int_{s_{0}}^{s} N_{r}^{2}\left(S_{\sigma}(s-\tau)\left(\left(1+e^{-\tau / 2}\right) \chi_{\varepsilon_{0}}(., \tau)\right)\right) d \tau \\
& -2 \int_{s_{0}}^{s} N_{r}^{2}\left(S_{\sigma}(s-\tau)\left(\operatorname{div}\left(\nu \mid \partial_{y} \gamma\right)\right)\right) d \tau \\
= & J_{1}+J_{2}+J_{3}+J_{4}+J_{5}
\end{aligned}
$$

Arguing as in Part 4 and using (83), we prove:

\section{Claim 2.23}

$$
\begin{aligned}
\left|J_{1}\right| \leq & C e^{\left(s-s_{0}\right)(1+\sigma)} e^{s_{0}(1-\varepsilon)} \\
\left|J_{2}\right| \leq & C \int_{s_{0}}^{s_{0}+\left(\left(s-R_{0}\right)-s_{0}\right)_{+}} \frac{e^{\left(s-\tau-R_{0}\right)(1+\sigma)}}{\left(1-e^{s-\tau-R_{0}}\right)^{1 / 20}}\left(L_{r}^{2}\left(Z(., s)^{2}\right)\right) d \tau \\
& +C e^{\left(s-s_{0}\right)(1+\sigma)} e^{s} \text { with } R_{0}=4 \varepsilon_{0}, \\
\left|J_{3}\right| \leq & C e^{\left(s-s_{0}\right)(1+\sigma)} e^{s}\left(1+e^{-s / 2}\right) \\
\left|J_{4}\right| \leq & C e^{\left(s-s_{0}\right)(1+\sigma)} e^{-\alpha e^{-s}} \text { where } \alpha>0, \\
\left|J_{5}\right| \leq & C e^{\left(s-s_{0}\right)(1+\sigma)} e^{-\beta e^{-s}} \text { where } \beta>0 .
\end{aligned}
$$

Proof: To estimate $J_{i=1,3,4}$, see page 1584 in [Vel92]. To treat $J_{2}$ and $J_{5}$, we proceed as in the proof of Lemma 2.14 of the previous part.

Summing up $J_{i=1 . .5}$, we obtain:

$$
\begin{aligned}
& N_{r}^{2}(Z(., s)) \leq \\
& C e^{\left(s-s_{0}\right)(1+\sigma)} e^{(1-\varepsilon) s}+C \int_{s_{0}}^{s_{0}+\left(\left(s-R_{0}\right)-s_{0}\right)+} \frac{e^{\left(s-\tau-R_{0}\right)(1+\sigma)}}{\left(1-e^{s-\tau-R_{0}}\right)^{1 / 20}}\left(L_{r}^{2}\left(Z(., s)^{2}\right)\right) d \tau,
\end{aligned}
$$

then using Proposition 2.17, we get $N_{r\left(s, s_{0}\right)}^{2}(Z(., s)) \leq C e^{\left(s-s_{0}\right)(1+\sigma)} e^{(1-\varepsilon) s}$ as $s \rightarrow-\infty$ for $s_{0} \leq s \leq \frac{s_{0}}{2}$. If we fix $s=s_{0} / 2$, then we obtain $N_{r\left(s, s_{0}\right)}^{2}(Z(., s)) \leq C e^{s(2(1-\varepsilon)-(1+\sigma))} \leq$ $C e^{s(1-(2 \varepsilon+\sigma))} \rightarrow 0$ as $s \rightarrow-\infty$, since $\epsilon$ is small enough and $\sigma=\frac{1}{100}$. This concludes the proof of Lemma 2.21.

As announced earlier, we don't give the proof of Lemma 2.22 and refer the reader to Part 4 and Section 2 from [Vel92]. This concludes the proof of Proposition 2.19 and rules out case (ii) of Proposition 2.7.

\section{Conclusion of Part 3 and the proof of the Liouville theorem}

We conclude from Part 4 and 5 that cases (ii) and (iii) of Proposition 2.7 are ruled out. By Part 3 , we obtain that $w \equiv \kappa$ or $w \equiv \varphi\left(s-s_{0}\right)$ for some real $s_{0}$, where $\varphi$ is defined in (12), which is the desired conclusion of Theorem 1. 


\section{Uniform bounds on $u(t)$ on quenching time and ODE type behavior}

We prove here Theorem 3. Let us first state the following convergence result which derives from [Guo90].

Proposition 4 (Guo) Consider $u(x, t)$ a type I solution of (1), which quenches in finite time T. Then, for any quenching point a

$$
\lim _{t \rightarrow T} u(a, t)(T-t)^{-\frac{1}{\beta+1}}=\kappa,
$$

Proof: See Theorem 3.10 page 77 in [Guo90]. Although Guo studied equation (1) on a finite interval with Dirichlet boundary conditions. One can easily check that this method holds on the whole space $\mathbb{R}$, whenever the solution is of type $\mathbf{I}$.

Consider $u(t)$ a nonnegative solution of type $\mathbf{I}$ of equation (1) that quenches at time $T$ and

$$
\left|\partial_{y} w(y, s)\right|+\frac{1}{w(y, s)} \leq M, \text { for all }(y, s) \in \mathbb{R} \times \mathbb{R}, \text { where } M>0 .
$$

In this section we proceed as in the proof of Theorem 1.1 in [MZ98a]. In the following, Part 1 is devoted to the proof of (i) of Theorem 3. In Part 2, we will prove (ii) of Theorem 3.

\section{Part 1: Uniform quenching estimates}

From the definition (6) and (10) of $w_{a}$ and $z_{a}$, it is enough to prove the second statement of i) for some $a \in \mathbb{R}$.

Consider $a$ a quenching point of $u$. For simplicity, we assume that $a=0$ and write $w$ (respectively $z$ ) instead of $w_{a}$ (respectively $z_{a}$ ).

We want to prove that $\inf _{y \in \mathbb{R}} w(y, s) \rightarrow \kappa$ and $\left\|\partial_{y} z(., s)\right\|_{L^{\infty}}+\left\|\partial_{y} z(., s)\right\|_{L^{\infty}} \rightarrow 0$ as $s \rightarrow+\infty($ where $z \equiv 1 / w)$.

Using Proposition 4, we know that $w(0, s) \rightarrow \kappa$ as $s \rightarrow+\infty$. Since $\inf _{y \in \mathbb{R}} w(y, s) \leq$ $w(0, s)$, we obtain

$$
\limsup _{s \rightarrow+\infty} \inf _{y \in \mathbb{R}} w(y, s) \leq \kappa
$$

and

$$
\liminf _{s \rightarrow+\infty}\|z(., s)\|_{L^{\infty}} \geq \kappa^{-1}
$$

hence,

$$
\liminf _{s \rightarrow+\infty}\|z(., s)\|_{L^{\infty}}+\left\|\partial_{y} z(., s)\right\|_{L^{\infty}}+\left\|\partial_{y}^{2} z(., s)\right\|_{L^{\infty}} \geq \kappa^{-1} .
$$

The conclusion follows if we prove that

$$
\limsup _{s \rightarrow+\infty}\|z(., s)\|_{L^{\infty}}+\left\|\partial_{y} z(., s)\right\|_{L^{\infty}}+\left\|\partial_{y}^{2} z(., s)\right\|_{L^{\infty}} \leq \kappa^{-1} .
$$

We argue by contradiction and assume that there exists a sequence $\left(s_{n}\right)_{n}$ such that $s_{n} \rightarrow+\infty$ as $n \rightarrow+\infty$ and

$$
\lim _{n \rightarrow+\infty}\left\|z\left(., s_{n}\right)\right\|_{L^{\infty}}+\left\|\partial_{y} z\left(., s_{n}\right)\right\|_{L^{\infty}}+\left\|\partial_{y}^{2} z\left(., s_{n}\right)\right\|_{L^{\infty}}=\kappa^{-1}+3 \varepsilon_{0},
$$


where $\varepsilon_{0}>0$. Up to extracting a subsequence, we have either

$\lim _{n \rightarrow+\infty}\left\|z\left(., s_{n}\right)\right\|_{L^{\infty}} \geq \kappa^{-1}+\varepsilon_{0}$, or $\lim _{n \rightarrow+\infty}\left\|\partial_{y} z\left(., s_{n}\right)\right\|_{L^{\infty}} \geq \varepsilon_{0}$, or $\lim _{n \rightarrow+\infty}\left\|\partial_{y y} z\left(., s_{n}\right)\right\|_{L^{\infty}} \geq \varepsilon_{0}$.

We deduce the existence of $\left(y_{n}^{i}\right)_{i=1 . .3}$ such that

$$
\begin{gathered}
\left\|z\left(., s_{n}\right)\right\|_{L^{\infty}}-\frac{\varepsilon_{0}}{2} \leq z\left(y_{n}^{1}, s_{n}\right), \\
\text { or }\left\|\partial_{y} z\left(., s_{n}\right)\right\|_{L^{\infty}}-\frac{\varepsilon_{0}}{2} \leq\left|\partial_{y} z\left(y_{n}^{2}, s_{n}\right)\right|, \\
\text { or }\left\|\partial_{y} z\left(., s_{n}\right)\right\|_{L^{\infty}}-\frac{\varepsilon_{0}}{2} \leq\left|\partial_{y}^{2} z\left(y_{n}^{3}, s_{n}\right)\right| .
\end{gathered}
$$

Introducing $y_{n}=y_{n}^{i}$, where $i=1,2$ or 3 , we define for each $n \in \mathbb{N}$

$$
W_{n}(y, s)=w\left(y+y_{n} e^{s / 2}, s+s_{n}\right) \text { and } Z_{n}(y, s)=1 / W_{n}(y, s) .
$$

We can see that $W_{n}$ satisfies (6) and $Z_{n}$ satisfies (10). More precisely, we claim the following:

Lemma 3.1 $\left(Z_{n}\right)_{n}$ is a sequence of solutions of (10) with the following properties:

i) $\lim _{n \rightarrow+\infty}\left|Z_{n}(0,0)\right| \geq \kappa^{-1}+\varepsilon_{0} / 2$, or $\lim _{n \rightarrow+\infty}\left|\partial_{y} Z_{n}(0,0)\right| \geq \varepsilon_{0} / 2$, or $\lim _{n \rightarrow+\infty}\left|\partial_{y}^{2} Z_{n}\right| \geq$ $\varepsilon_{0} / 2$.

ii) For all $R>0$, there exists $n_{0} \in \mathbb{N}$, such that for all $n \geq n_{0}$,

- $Z_{n}$ is defined for all $(y, s) \in[-R, R]^{2}$ and $W_{n}=1 / Z_{n}$ satisfies

$$
\left|\partial_{y} W_{n}(y, s)\right|+\frac{1}{W_{n}(y, s)} \leq M
$$

- $Z_{n} \geq 0$ and $\left\|Z_{n}(., s)\right\|_{L^{\infty}\left([-R, R]^{2}\right)}+\left\|\partial_{y} Z_{n}(., s)\right\|_{L^{\infty}\left([-R, R]^{2}\right)} \leq M$.

- There exists $m(R)>0$ such that $\left\|Z_{n}(., s)\right\|_{C^{2,1}\left([-R, R]^{2}\right)} \leq m(R)$, where $C^{2,1}\left([-R, R]^{2}\right)$ stands for the functions which are continuously differentiable twice in space and once in time.

Proof of Lemma 3.1: We give the following version of Lemma 2.1,

Claim 3.2 (Parabolic estimates) For all $(y, s) \in[-R, R]^{2}$ :

(i) $\left|Z_{n}(y, s)\right|+\left|\partial_{y} Z_{n}(y, s)\right|+\left|\partial_{y}^{2} Z_{n}(y, s)\right| \leq M$ and $\left|\partial_{s} Z_{n}\right| \leq M(1+|y|)$.

(ii) For all $R>0, Z_{n}, \partial_{y} Z_{n}(y, s), \partial_{y}^{2} Z_{n}$ and $\partial_{s} Z_{n}$ are bounded in $C_{\alpha}^{0}\left([-R, R]^{2}\right)$ for some $\alpha \in(0,1)$, where

$$
C_{\alpha}^{0}\left([-R, R]^{2}\right)=\left\{\psi \in L^{\infty}\left[(-R, R)^{2}\right] \mid \sup _{(\xi, \tau),\left(\xi^{\prime}, \tau^{\prime}\right) \in[-R, R]^{2}} \frac{\left|\psi(\xi, \tau)-\psi\left(\xi^{\prime}, \tau^{\prime}\right)\right|}{\left(\left|\xi-\xi^{\prime}\right|+\left|\tau-\tau^{\prime}\right|^{1 / 2}\right)^{\alpha}}<\infty\right\}
$$

Using the fact that $u$ is of type $\mathbf{I}$ and claim 3.2, we proceed as in the proof of Lemma 2.2 in [MZ98a].

Now, using the compactness property of $\left(Z_{n}\right)$, shown in Lemma 3.1, we find $Z_{R} \in$ $C^{2,1}\left([-R, R]^{2}\right)$ such that up to extracting a subsequence, $Z_{n} \rightarrow Z_{R} \in C^{2,1}\left([-R, R]^{2}\right)$. Using a diagonal process, we find $Z \in C^{2,1}\left(\mathbb{R}^{2}\right)$ such that $Z_{n} \rightarrow Z$ in $\in C_{\text {loc }}^{2,1}\left(\mathbb{R}^{2}\right)$. From Lemma 3.1, we have 
(a) $Z$ satisfies (10) for $(y, s) \in \mathbb{R} \times \mathbb{R}$.

(b) $Z \geq 0$ and $\|Z\|_{L^{\infty}(\mathbb{R} \times \mathbb{R})}+\left\|\partial_{y} Z\right\|_{L^{\infty}(\mathbb{R} \times \mathbb{R})} \leq C$.

(c) $Z(0,0) \geq \kappa^{-1}+\varepsilon_{0} / 2$, or $\left|\partial_{y} Z(0,0)\right| \geq \varepsilon_{0} / 2$ or $\left|\partial_{y}^{2} Z(0,0)\right| \geq \varepsilon_{0} / 2$.

Now, we have to consider two cases:

Case 1: If there exits $\left(y_{0}, s_{0}\right)$ such that $Z\left(y_{0}, s_{0}\right)=0$. Then, doing as in the case 1 of proof of Lemma 2.4, we obtain $Z \equiv 0$, which is impossible because of (c).

Case 2: For all $(y, s), Z(y, s)>0$. In this case, we have $W_{n}=1 / Z_{n} \rightarrow W=1 / V$ in $C_{l o c}^{2,1}\left(\mathbb{R}^{2}\right)$ and $\left|\partial_{y} Z_{n}(0,0)\right|^{2} / Z_{n}(0,0) \rightarrow\left|\partial_{y} Z(0,0)\right|^{2} / Z(0,0)$.

Since $W_{n}$ satisfies (1), the same holds for $W$. Using ii) of claim 3.2 , we see that

$$
\forall(y, s) \in \mathbb{R}^{2} \quad\left|\partial_{y} W(y, s)\right|+\frac{1}{W(y, s)} \leq M .
$$

Using our Liouville theorem, we get $W \equiv \kappa$ or $W(y, s)=\varphi\left(s-s_{0}\right)$, where $\varphi\left(s-s_{0}\right)=$ $\kappa\left(1+e^{s}\right)^{1 /(\beta+1)}$. In all cases, this contradicts (c). This concludes the proof of $\mathrm{i}$ ) of Theorem 3 .

Part 2: ODE type behavior

Consider a type $\mathbf{I}$ solution $u(t)$ of $(1)$, that quenches in finite time $T>0$, with initial condition satisfying (2). We know from (88) that:

$$
\forall t \in[0, T), x \in \mathbb{R},\left|u_{x}(x, t)\right|(T-t)^{\frac{1}{2}-\frac{1}{\beta+1}}+\frac{1}{u(x, t)}(T-t)^{\frac{1}{\beta+1}} \leq M, \text { where } M>0 .
$$

Let us prove now the uniform pointwise control of the diffusion term by the nonlinear term, which asserts that the solution $u(t)$ behaves everywhere like the ODE $u^{\prime}=-u^{-\beta}$ (up to a constant).

Since $u_{0} \in L^{\infty}$, hence

$$
\|u(t, .)\|_{L^{\infty}} \leq\left\|u_{0}\right\|_{L^{\infty}}
$$

from equation (1), it is enough to prove that

$$
\begin{aligned}
& \forall \varepsilon, \quad \exists c_{\varepsilon}>0 \text { such that for all }(x, t) \in \mathbb{R} \times[T / 2, T), \\
& \qquad\left|\partial_{t} v-v^{2+\beta}\right| \leq \varepsilon v^{2+\beta}+c_{\varepsilon}, \text { where } v=1 / u .
\end{aligned}
$$

The plan of the proof is the same as in [MZ98a] and [MZ00]. However, the Giga-Kohn property "small local energy implies no blow-up locally" breaks down. We proceed as in [NZ10], where the authors proved that "small $L_{\rho}^{2}$ norm implies no blow-up locally". In our case we have the following Lemma:

Lemma 3.3 For all $M>0$, there exist positive $\epsilon_{0}, C_{0}$ and $M_{0}$ such that if $U$ is a type $\boldsymbol{I}$ solution of (1) and

$$
\forall\left|x_{0}\right| \leq 1,\left\|Z_{x_{0}}(., 0)\right\|_{L_{\rho}^{2}} \leq \epsilon
$$

for some $0<\epsilon \leq \eta_{0}$, where

$$
y=\frac{x-x_{0}}{\sqrt{1-t}}, s=-\log (1-t), Z_{x_{0}}(y, s)=(1-t)^{\frac{1}{\beta+1}} \frac{1}{U(x, t)},
$$


then:

(i) For all $\left|x_{0}\right| \leq 1$ and $s \in[0,+\infty)$,

$$
\left\|Z_{x_{0}}(., s)\right\|_{L_{\rho}^{2}} \leq C_{0} \epsilon e^{-\frac{s}{\beta+1}} .
$$

(ii) For all $|x| \leq 1$ and $t \in[0,1)$, we have $|U(x, t)| \geq M_{0}$.

Proof: The idea of the proof is the same as in the proof of Proposition 3.3 in [NZ10]. (i) For simplicity, we write $Z$ instead of $Z_{x_{0}}$. Since $Z$ is a solution of (10), we multiply (10) by $Z \rho$ and integrate to get

$$
I^{\prime}(s) \leq-\int-\frac{2}{\beta+1} I(s)+2 \int|Z(y, s)|^{\beta+3} \rho(y) d y, \text { where } I(s)=\int Z(y, s)^{2} \rho(y) d y .
$$

We note by $\left(\partial_{y} Z\right)^{2} / Z=\left(\partial_{y} w\right)^{2} Z^{3},(10)$ and (91) that $Z$ satisfies

$$
\partial_{s} Z \leq\left(\partial_{y}^{2} Z-\frac{1}{2} y \cdot \partial_{y} Z+Z\right)+\sigma Z, \text { where } \sigma \text { is a positive constant. }
$$

Using Lemma 2.9 (for $Z$ ), we see that there exist $C^{*}(M)>0$ and $s^{*}=s^{*}(p+1)>0$ such that for all $s \geq s^{*}$

$$
\int|Z(y, s)|^{\beta+3} \rho(y) d y \leq C^{*}\left\|Z\left(., s-s^{*}\right)^{2}\right\|_{L_{\rho}^{2}}^{\frac{\beta+3}{2}}
$$

Now, we divide the proof in two steps:

Step 1: $0 \leq s \leq s^{*}$. Using (94) and the fact that $Z$ is bounded by $M>0$ (see (92)), we get

$$
I^{\prime}(s) \leq \lambda I(s) \text { for some } \lambda=\lambda(M)>0,
$$

hence $I(s) \leq e^{\lambda s} I(0) \leq e^{\lambda s} \epsilon^{2} \leq \frac{C_{0}^{2}}{2} \epsilon^{2} e^{-\frac{2 s}{\beta+1}}$, where we define $C_{0}^{2}=2 e^{\left(\lambda+\frac{2}{\beta+1}\right) s^{*}}$. This gives (93) for $0 \leq s \leq s^{*}$.

Step 2: $s \geq s^{*}$. In this step, we argue by contradiction to prove (93) for all $s \geq s^{*}$. We suppose that there exists $s_{1}>s^{*}$, such that

$$
\begin{aligned}
I(s) & <\left(C_{0} \epsilon\right)^{2} e^{-\frac{2 s}{\beta+1}}, \text { for all } s^{*} \leq s<s_{1} \\
I\left(s_{1}\right) & =\left(C_{0} \epsilon\right)^{2} e^{-\frac{2 s_{1}}{\beta+1}} .
\end{aligned}
$$

Let $F(s)=I(s)\left(C_{0} \epsilon\right)^{-2} e^{\frac{2 s}{\beta+1}}$. From (94), (95), (96) and Step 1, we have for all $s^{*} \leq s \leq s_{1}$,

$$
\begin{aligned}
F^{\prime}(s) & \leq C^{*}\left(C_{0} \epsilon\right)^{-2} e^{\frac{2 s}{\beta+1}} I\left(s-s^{*}\right)^{\frac{\beta+3}{2}} \\
& \leq C^{*}\left(C_{0} \epsilon\right)^{\beta+1} e^{\frac{2 s}{\beta+1}} e^{-\left(s-s^{*}\right) \frac{\beta+3}{\beta+1}} \leq C^{*}\left(C_{0} \epsilon\right)^{\beta-1} e^{\frac{\beta+3}{\beta+1} s^{*}} e^{-s} .
\end{aligned}
$$

Since $F\left(s^{*}\right) \leq \frac{1}{2}$ from the step above, we integrate the last inequality to obtain

$$
\begin{aligned}
F\left(s_{1}\right) & \leq C^{*}(C \epsilon)^{\beta-1} e^{s^{*}}\left(e^{-s^{*}}-e^{-s_{1}}\right)+F\left(s^{*}\right), \\
& \leq C^{*}(C \epsilon)^{\beta-1} e^{\frac{2 s^{*}}{\beta+1}}+\frac{1}{2} \leq \frac{3}{4},
\end{aligned}
$$


for $\epsilon \leq \epsilon_{0}(M)$ small enough. This contradicts (97). Therefore, (93) holds.

(ii) Applying parabolic regularity to equation (6) and using estimate (92), we get for all $\left|x_{0}\right| \leq 1, R>0$ and $|y|<R,\left|Z_{x_{0}}(y, s)\right| \leq M_{0} e^{-\frac{s}{\beta+1}}$, hence for all $t \in[0,1),\left|U\left(x_{0}, t\right)\right| \geq$ $M_{0}$, for some $M_{0}=M_{0}(M)$. This ends the proof of Lemma 3.3.

We argue by contradiction and assume that for some $\varepsilon_{0}>0$, there exists $\left(x_{n}, t_{n}\right)_{n \in \mathbb{N}}$, a sequence of elements of $\mathbb{R} \times\left[\frac{T}{2}, T\right)$, such that

$$
\forall n \in \mathbb{N},\left|\partial_{x}^{2} v\left(x_{n}, t_{n}\right)\right| \geq \varepsilon_{0}\left|v\left(x_{n}, t_{n}\right)\right|^{2+\beta}+n .
$$

From the uniform estimates of i) of Theorem 3 and the parabolic regularity, since $\left\|\partial_{x x} v(., t)\right\|_{L^{\infty}}$ is bounded on compact sets of $\left[\frac{T}{2}, T\right)$, we have

$$
T-t_{n} \rightarrow 0, \text { as } n \rightarrow \infty .
$$

We note that i) of Theorem 3 implies that $v\left(x_{n}, t_{n}\right)\left(T-t_{n}\right)^{\frac{1}{\beta+1}}$ is uniformly bounded, therefore, we can assume that it converges as $n \rightarrow+\infty$. Let us consider two cases:

i) Estimates in the very singular region: $v\left(x_{n}, t_{n}\right)\left(T-t_{n}\right)^{\frac{1}{\beta+1}} \rightarrow \kappa_{0}>0$. From (98), it follows that

$$
\left\|\partial_{x}^{2} v\left(., t_{n}\right)\right\|_{L^{\infty}} \geq\left|\partial_{x}^{2} v\left(x_{n}, t_{n}\right)\right| \geq \varepsilon_{0}\left(\frac{\kappa_{0}}{2}\right)^{2+\beta}\left(T-t_{n}\right)^{-\frac{\beta+2}{\beta+1}},
$$

with $t_{n} \rightarrow T$, which contradicts i) Theorem 3 .

ii) Estimates in the singular region: $v\left(x_{n}, t_{n}\right)\left(T-t_{n}\right)^{\frac{1}{\beta+1}} \rightarrow 0$.

We consider $n$ large enough, such that

$$
v\left(x_{n}, t_{n}\right)\left(T-t_{n}\right)^{\frac{1}{\beta+1}} \leq \eta_{0} / 3 \text {, where } \eta_{0} \text { is defined in Lemma } 3.3 .
$$

We take $t_{n}^{0} \rightarrow T$ such that

$$
\left(T-t_{n}^{0}\right)^{-\frac{\beta+2}{\beta+1}}=\sqrt{n} .
$$

Using (98) and i) of Theorem 3, we obtain:

$$
n \leq\left|\partial_{x}^{2} v\left(x_{n}, t_{n}\right)\right| \leq C_{0}\left(T-t_{n}\right)^{-\frac{\beta+2}{\beta+1}},
$$

hence $t_{n}^{0}<t_{n}$. Now we distinguish two cases:

Case 1. We assume that (up to extracting a subsequence) there exists $t_{n}^{\prime} \in\left(t_{n}^{0}, t_{n}\right)$, such that $v\left(x_{n}, t_{n}^{\prime}\right)\left(T-t_{n}^{\prime}\right)^{\frac{1}{\beta+1}}=\frac{2}{3} \eta_{0}$. If we consider

$$
v_{n}(\xi, \tau)=\left(T-t_{n}^{\prime}\right)^{\frac{1}{\beta+1}} v\left(x_{n}+\xi \sqrt{T-t_{n}^{\prime}}, t_{n}^{\prime}+\tau\left(T-t_{n}^{\prime}\right)\right),
$$

then, we have from i) of Theorem 3 ,

$$
v_{n}(0,0)=\frac{2}{3} \eta_{0},\left\|\partial_{\xi} v_{n}(., 0)\right\|_{L^{\infty}}+\left\|\partial_{\xi}^{2} v_{n}(., 0)\right\|_{L^{\infty}} \rightarrow 0 .
$$

If we note by $u_{n}=1 / v_{n}$, then we have from (2) and (91):

$\forall \tau<1, \xi \in \mathbb{R},\left|\partial_{\xi} u_{n}(\xi, \tau)\right|(1-\tau)^{\frac{1}{2}-\frac{1}{\beta+1}}+\frac{1}{u_{n}(\xi, \tau)}(1-\tau)^{\frac{1}{\beta+1}} \leq M$ and $\partial_{\tau} u_{n}=\partial_{x}^{2} u_{n}-u_{n}^{-\beta}$. 
Then, we can see that $v_{n}$ is bounded and satisfies (8). Using parabolic regularity, we can extract a subsequence (still denoted by $t_{n}$ ) such that, $v_{n}(\xi, \tau) \rightarrow \hat{v}(\xi, \tau)$ in $\mathcal{C}^{2,1}$ of every compact set of $\mathbb{R} \times(-\infty, 1)$. We note that if there exists $\left(\xi_{0}, \tau_{0}\right)$ such that $\hat{v}\left(\xi_{0}, \tau_{0}\right)=0$, then $\hat{v} \equiv 0$ (see case 1 in the proof of Lemma 2.4). This case is imposible because $\hat{v}(0,0)=3 / 2 \eta_{0}$. Therefore, it holds that for all $(\xi, \tau), \hat{v}(\xi, \tau)>0$. Recalling that $u_{n}(\xi, \tau)=1 / v_{n}(\xi, \tau)$, we have

$$
u_{n}(\xi, \tau)=1 / v_{n}(\xi, \tau) \rightarrow \hat{u}(\xi, \tau)=1 / \hat{v}(\xi, \tau) \text { in } \mathcal{C}^{2,1} \text { of every compact set of } \mathbb{R} \times(-\infty, 1)
$$

and by (102), we get

$$
\partial_{\tau} \hat{u}=\partial_{\xi}^{2} \hat{u}-\hat{u}^{-\beta}, \hat{u}(0,0)=\frac{3}{2 \eta_{0}} \text { and }\left|\partial_{\xi} \hat{u}(\xi, \tau)\right|(1-\tau)^{\frac{1}{2}-\frac{1}{\beta+1}}+\frac{1}{\hat{u}(\xi, \tau)}(1-\tau)^{\frac{1}{\beta+1}} \leq M .
$$

Using the Liouville Theorem (see Theorem 1), we get

$$
\hat{u}(\xi, \tau)=\kappa\left(\left(\frac{3 \kappa}{2 \eta_{0}}\right)^{-(\beta+1)}-\tau\right)^{\frac{1}{\beta+1}} .
$$

We claim that it is enough to prove that the convergence of $u_{n} \rightarrow \hat{u}$ takes place in $C^{2,1}((-1,1) \times[0,1))$ to conclude. Indeed, if we have this extended convergence, then we write from (98) and the definition (100) of $v_{n}$,

$$
\begin{aligned}
\left|\partial_{\xi}^{2} v_{n}\left(0, \tau_{n}\right)\right| & =\left(T-t_{n}^{\prime}\right)^{\frac{\beta+2}{\beta+1}}\left|\partial_{\xi \xi} v\left(0, t_{n}\right)\right| \\
& \geq \varepsilon_{0}\left|v\left(0, t_{n}\right)\right|^{\beta+2}\left(T-t_{n}^{\prime}\right)^{\frac{\beta+2}{\beta+1}} \\
& =\varepsilon_{0}\left|v_{n}\left(0, \tau_{n}\right)\right|^{2+\beta} \\
& \geq \varepsilon_{0} \min _{\tau \in[0,1]}\left|v_{n}(0, \tau)\right|^{2+\beta}
\end{aligned}
$$

with $\tau_{n}=\frac{t_{n}-t_{n}^{\prime}}{T-t_{n}^{\prime}}$. Letting $n \rightarrow \infty$, we obtain

$$
\partial_{\xi}^{2} v_{n}\left(0, \tau_{n}\right) \rightarrow 0 \text { and } \min _{\tau \in[0,1]}\left|v_{n}(0, \tau)\right|^{2+\beta} \rightarrow \min _{\tau \in[0,1]}|\hat{v}(\tau)|^{2+\beta} .
$$

Hence

$$
0 \geq \varepsilon_{0} \min _{\tau \in[0,1]}|\hat{v}(\tau)|^{2+\beta}=\varepsilon_{0}|\hat{0}(\tau)|^{2+\beta}>0
$$

which is a contradiction.

Let us then extend the convergence. If we consider the following similarity variables,

$$
y=\frac{\xi-\xi_{0}}{\sqrt{1-\tau}}, s=-\log (1-\tau), Z_{n, \xi_{0}}(y, s)=(1-\tau)^{-\frac{1}{\beta+1}} v_{n}(\xi, \tau),
$$

then, we see from (101) that for all $\left|\xi_{0}\right| \leq 1,\left\|Z_{n, \xi_{0}}(., 0)\right\|_{L_{\rho}^{2}} \leq \eta_{0}$, for $n$ large enough. Using Lemma 3.3, we get for all $|\xi| \leq 1$ and $\tau \in[0,1),\left|v_{n}(\xi, \tau)\right| \leq \frac{1}{M_{0}}$. Using the parabolic regularity, we can extend the convergence, and then reach the contradiction (103). This concludes Case 1.

Case 2. We assume that for some $n_{0} \in \mathbb{N}$, for all $n \geq n_{0}$ and $t \in\left[t_{n}^{0}, t_{n}\right]$, we have:

$$
v\left(x_{n}, t\right)(T-t)^{\frac{1}{\beta+1}}<\frac{2}{3} \eta_{0} .
$$


Then, we take $t_{n}^{\prime}=t_{n}^{0}$ and introduce $v_{n}$ by (100). As in Case 1 , we obtain by Lemma 3.3 and the parabolic regularity:

$$
\forall|\xi| \leq 1 \text { and } \tau \in[0,1),\left|v_{n}(\xi, \tau)\right| \leq 1 / M_{0}, \quad\left|\partial_{\xi}^{2} v_{n}\left(0, \tau_{n}\right)\right| \leq C_{0} \eta_{0} \text { where } \tau_{n}=\frac{t_{n}-t_{n}^{0}}{T-t_{n}^{0}} .
$$

Therefore, we get from (98), (100) and (99):

$$
n \leq\left|\partial_{\xi}^{2} v_{n}\left(x_{n}, t_{n}\right)\right|=\left(T-t_{n}^{0}\right)^{-\frac{\beta+2}{\beta+1}}\left|\partial_{\xi}^{2} v_{n}\left(0, \tau_{n}\right)\right| \leq_{0} \eta_{0}\left(T-t_{n}^{0}\right)^{-\frac{\beta+2}{\beta+1}}=C_{0} \eta_{0} \sqrt{n},
$$

which is a contradiction, as $n \rightarrow \infty$. This ends Case 2 and concludes the proof of Theorem 3 .

\section{A Proof of Proposition 2.7}

We proceed as in Appendix A [MZ98a], the most important difference, is that in our case we have not $w$ bounded. Let us introduce some notations,

$$
v_{+}(y, s)=v_{0}(s) h_{0}(y)+v_{1}(s) h_{1}(y), \quad v_{\text {null }}(y, s)=v_{2}(s) h_{2}(y) .
$$

We divide the proof in two parts: in part 1, we show that either $v_{\text {null }}$ or $v_{+}$is predominant in $L_{\rho}^{2}$ as $s \rightarrow-\infty$. In part 2 , we show that in the case where $v_{+}$is predominant, then either $v_{0}(s)$ or $v_{1}(s)$ predominates the other.

Step 1 of the Proof: Competition between $v_{+}, v_{n u l l}$ and $v_{-}$ First, we recall from (38)

$$
\forall(y, s) \in \mathbb{R}^{2}, \partial_{s} v=\mathcal{L} v-f(v) .
$$

Let us introduce some notations

$$
z(s)=\left\|v_{+}(., s)\right\|_{L_{\rho}^{2}} \quad, x(s)=\left\|v_{\text {null }}(., s)\right\|_{L_{\rho}^{2}} \text { and } y(s)=\left\|v_{-}(., s)\right\|_{L_{\rho}^{2}} .
$$

Projection (105) onto the unstable subspace of $\mathcal{L}$ forming the $L_{\rho}^{2}$-inner product with $v_{+}$, and using standard inequalities, Lemma 2.6 and (40). We get

$$
\dot{z} \geq \frac{1}{2} z-N \text {, where } N=\left\|v^{2}\right\|_{L_{\rho}^{2}} .
$$

Working similarly with $v_{\text {null }}$ and $v_{-}$we arrive at the system

$$
\begin{aligned}
& \dot{z} \geq \frac{1}{2} z-N \\
& |\dot{x}| \leq N, \\
& \dot{y} \leq-\frac{1}{2} y+N .
\end{aligned}
$$

If we knew for $|s|$ large enough

$$
N \leq \varepsilon(x+y+z),
$$

we could use ODE techniques to conclude the step. However, we do not have this information at this stage. We thus estimate $N$ proceeding as in Section 4 in [FK92b]. We 
note that in [FK92b] (107) was proved under the additional assumption that $v(y, s)$ is uniformly bounded. One can check in [FK92b] that this assumption is only used for the derivation of the estimate $|f(v)| \leq c|v|$, which in our case is true by (40). Then, we get by (107) and (106),

$$
\begin{aligned}
& \dot{z} \geq\left(\frac{1}{2}-\varepsilon\right) z-\varepsilon(x+y) \\
& |\dot{x}| \leq \varepsilon(x+y+z), \\
& \dot{y} \leq-\left(\frac{1}{2}+\varepsilon\right) y+\varepsilon(x+z) .
\end{aligned}
$$

Now, let us recall Lemma A.1 from [NZ10].

Lemma A.1 (Merle-Zaag) Let $x(s), y(s)$, and $z(s)$ be absolutely continuous, real-valued functions that are nonnegative and satisfy

(i) $(x, y, z)(s) \rightarrow 0$ as $s \rightarrow \infty$, and $\forall s \leq s^{\star}, x(s)+y(s)+z(s) \neq 0$, and (ii) $\forall \varepsilon>0, \exists s_{0} \in \mathbb{R}$ such that $\forall s \leq s_{0}$

$$
\left\{\begin{array}{l}
\dot{z} \geq c_{0} z-\varepsilon(x+y) \\
|\dot{x}| \leq \varepsilon(x+y+z) \\
\dot{y} \leq-c_{0} y+\varepsilon(x+y)
\end{array}\right.
$$

Then, either $x+y=o(z)$ or $y+z=o(x)$ as $s \rightarrow-\infty$.

Applying Lemma above to (108), we get either

$$
\left\|v_{-}(., s)\right\|_{L_{\rho}^{2}}+\left\|v_{+}(., s)\right\|_{L_{\rho}^{2}}=o\left(\left\|v_{\text {null }}(., s)\right\|_{L_{\rho}^{2}}\right),
$$

or

$$
\left\|v_{-}(s)\right\|_{L_{\rho}^{2}}+\left\|v_{\text {null }}(., s)\right\|_{L_{\rho}^{2}}=o\left(\left\|v_{+}(., s)\right\|_{L_{\rho}^{2}}\right) .
$$

Step 2 of the Proof: Competition between $v_{0}$ and $v_{1}$

In this step we focus on the case where $\left\|v_{-}(., s)\right\|_{L_{\rho}^{2}}+\left\|v_{\text {null }}(., s)\right\|_{L_{\rho}^{2}}=o\left(\left\|v_{+}(., s)\right\|_{L_{\rho}^{2}}\right)$. We will show that it leads to either case (ii) or (iii) of Proposition 2.7. We want to derive from (105) the equations satisfied by $v_{0}$ and $v_{1}$. For this we will estimate in the following $\int f(v) k_{m}(y) \rho(y) d y$, for $m=0,1$ where

$$
k_{m}(y)=h_{m}(y) /\left\|h_{m}(y)\right\|_{L_{\rho}^{2}}^{2} .
$$

Lemma A.2 There is $0>0$ and an integer $k^{\prime}>4$ such that for all $\delta \in\left(0, \delta_{0}\right), \exists s_{0} \in \mathbb{R}$ such that $\forall s \leq s_{0}, \int v^{2}|y|^{k^{\prime}} \rho d y \leq c_{0}\left(k^{\prime}\right) \delta^{4-k^{\prime}} z(s)^{2}$

Poof: This lemma is analogous to Lemma A.3 p 175 from [MZ98a], it have been proved under the additional assumption that $v(y, s)$ is uniformly bounded. One can check that this assumption is only used for the derivation of the estimate $f(v)<C v$, which is in our case true.

Proceeding as in Appendix A from [MZ98a] and doing the projection of equation (105) respectively on $k_{0}(y)$ and $k_{1}(y)$, we obtain

$$
v_{0}^{\prime}(s)=v_{0}(s)-\frac{\beta}{2 \kappa} z(s)^{2}(1+\alpha(s))
$$


Using the same type of calculations as for $\int v^{3} \rho d y$ and Lemma A.2, we can prove that $\int v^{2} k_{1}(y) \rho d y=O\left(z(s)^{2}\right)$. Therefore, (105) yields the following vectorial equation:

$$
v_{1}^{\prime}(s)=\frac{1}{2} v_{1}(s)+\gamma(s) z(s)^{2},
$$

where $\gamma$ is bounded.

Proceeding as in [MZ98a], we get:

$$
\forall \varepsilon>0, v_{0}(s)=O\left(e^{(1-\varepsilon) s}\right) \text { and } v_{1}(s)=C_{1} e^{\frac{s}{2}}+O\left(e^{(1-\varepsilon) s}\right)
$$

and

$$
\forall \varepsilon>0, v_{0}(s)=-\frac{\beta}{\kappa}\left|C_{1}\right|^{2} s e^{s}(1+o(1))+C_{0} e^{s}+O\left(e^{2(1-\varepsilon)}\right) \text { as } s \rightarrow-\infty
$$

Two cases then arise:

- If $C_{1} \neq 0$, then $\left.v_{1}(s) \equiv C_{1} e^{\frac{s}{2}} \gg v_{0}(s)\right) O\left(s e^{s}\right)$, from (112). We note that applying Lemma 2.9 to $|v|$, we have for $s$ large enough (and $s<0$ ),

$$
N^{2}=\int|v(y, s)|^{4} \rho(y) d y \leq C^{*}\|v(., s-s *)\|_{L_{\rho}^{2}}^{2},
$$

for some positive $s *$ and $C^{*}$.

Recalling (105) and using (113), we obtain $\dot{y} \leq-\frac{1}{2} y+c^{*}\|v(., s-s *)\|_{L_{\rho}^{2}}^{2} \leq-\frac{1}{2} y+$ $c e^{s}$. Then, we obtain $x=\left\|v_{\text {null }}(., s)\right\|_{L_{\rho}^{2}}=O\left(e^{s}\right)$. We conclude that $\| v(., s)-$ $C_{1} e^{s / 2} y \|_{L_{\rho}^{2}}=O\left(e^{s(1-\varepsilon)}\right)$ as $s \rightarrow-\infty$, for some $\varepsilon>0$. This is case (ii) of Proposition 2.7 .

- If $C_{1}=0$, we obtain case (iii) of Proposition 2.7. Indeed, let us first improve the estimate of $v$. In fact, from (112) we have $v_{0}=C_{0} e^{s}+O\left(e^{3 / 2 s}\right)$ and from (111) $v_{1}=O\left(e^{3 / 2 s}\right)$.

Recalling (105) and using (113), we obtain $\dot{y} \leq-\frac{1}{2} y+c^{*}\|v(., s-s *)\|_{L_{\rho}^{2}}^{2} \leq-\frac{1}{2} y+c e^{2 s}$. Then, we have $y=O\left(e^{3 / 2 s}\right)$. Similarly, we obtain that $x=O\left(e^{3 / 2 s}\right)$ and we conclude that

$$
\left\|v(., s)-v_{0}(s)\right\|_{L_{\rho}^{2}}=O\left(e^{3 / 2 s}\right) .
$$

This is case (iii) of Proposition 2.7.

\section{Step 3: Case where $v_{\text {null }}$ dominates}

In the following, we prove that (iii) of Proposition 2.7 holds. First, we prove the following Lemma:

Lemma A.3 Assume that

$$
\left\|v_{+}(., s)\right\|_{L_{\rho}^{2}}+\left\|v_{-}(., s)\right\|_{L_{\rho}^{2}}=o\left(\left\|v_{\text {null }}(., s)\right\|_{\left.L_{\rho}^{2}\right)}\right.
$$

holds, then

$$
v(y, s)=\frac{\kappa}{4 \beta s}\left(y^{2}-2\right)+o\left(\frac{1}{s}\right),
$$

in $L_{\rho}^{2}$ as $s \rightarrow-\infty$. 
Proof: Since $v_{\text {null }}(y, s)=v_{2}(s) h_{2}(y)$, we note that $v_{2}(s)=\int v k_{2}(y) \rho$. Using (41), we rewrite equation (105) as follows

$$
v_{s}=\mathcal{L} v-\frac{\beta}{2 \kappa} v^{2}+g(v)
$$

where $g(v)=O\left(v^{3}\right)$. Projecting equation (115) onto $h_{2}(y)$ we get

$$
\begin{aligned}
\partial_{s} v_{2} & =-\frac{\beta}{2 \kappa} \int v^{2} k_{2}(y) \rho(y)+\int g(v) k_{2}(y) \rho(y) \\
& =-\frac{\beta}{2 \kappa} \int v_{\text {null }}^{2} k_{2}(y) \rho(y)+\frac{\beta}{2 \kappa} \int\left(v_{\text {null }}^{2}-v^{2}\right) k_{2}(y) \rho(y)+\int g(v) k_{2}(y) \rho(y) \\
& =-\frac{\beta}{2 \kappa} 8 v_{2}^{2}+\frac{\beta}{2 \kappa} \mathcal{E}_{1}+\mathcal{E}_{2},
\end{aligned}
$$

where we use the fact that $\int v_{n u l l} k_{2} \rho=v_{2}^{2} \int h_{2}^{2} k_{2} \rho=8 v_{2}^{2}$. we next estimate $\mathcal{E}_{1}$ and $\mathcal{E}_{2}$. For this we need the following lemma

Lemma A.4 There exists $\alpha_{0}>0$ and an integer $k^{\prime}>4$ such that for all $\alpha \in\left(0, \alpha_{0}\right)$, there exists $s_{0} \in \mathbb{R}$ such that for all $s \leq s_{0}$,

$$
\int|v|^{2}|y|^{k^{\prime}} \rho d y \leq c_{0}\left(k^{\prime}\right) \alpha^{4-k^{\prime}} \int v_{n u l l}^{2} \rho d y \text {. }
$$

Proof: See Lemma C.1 in [MZ98a] (page 187)

Recalling that $v=v_{+}+v_{\text {null }}+v_{-}$, we write on the one hand:

$$
\begin{aligned}
\left|\mathcal{E}_{1}\right| & \leq \int\left|v_{+}+v_{-}\right| \times\left|v+v_{\text {null }}\right|\left|k_{2}(y)\right| \rho, \\
& \leq c\left(\int\left|v_{+}+v_{-}\right|^{2} \rho\right)^{1 / 2}\left\{\left(v^{2} k_{2}^{2}(y) \rho\right)^{1 / 2}+\left(\int v_{\text {null }}^{2} k_{2}^{2} \rho\right)^{1 / 2}\right\} .
\end{aligned}
$$

We know by (114) $\left(\int\left|v_{+}+v_{-}\right|^{2} \rho\right)^{1 / 2}=o\left(v_{2}\right)$ and

$$
\left(v^{2} k_{2}^{2}(y) \rho\right)^{1 / 2}+\left(\int v_{\text {null }}^{2} k_{2}^{2} \rho\right)^{1 / 2} \leq \underbrace{\left(v^{2} k_{2}^{2}(y) \rho\right)^{1 / 2}}_{I}+c\left|v_{2}\right| .
$$

To treat $I$, we have from Lemma A.4

$$
\int v^{2} k_{2}^{2} \rho \leq c \int|v|^{2} \rho+\int|v|^{2}|y|^{k^{\prime}} \rho \leq c \in v^{2} \rho \leq c v_{2}^{2},
$$

we conclude that $\mathcal{E}_{1}=o\left(v_{2}^{2}\right)$. It remains to estimate $\mathcal{E}_{1}$, we consider $\alpha \in\left(0, \alpha_{0}\right)$ and we proceed as in Appendix C from [MZ98a], (page 189). We write for $m=0$ or $m=2$ :

$$
\begin{aligned}
\int|v|^{3}|y|^{m} \rho d y & \leq \int_{|y| \leq \alpha^{-1}}|v|^{3}|y|^{m} \rho d y+\int_{|y| \geq \alpha^{-1}}|v|^{3}|y|^{m} \rho \\
& \leq \varepsilon \alpha^{-m} \int_{|y| \leq \alpha^{-1}}|v|^{2} \rho d y+C M \alpha^{k^{\prime}-m-1}(1+\alpha) \int_{|y| \geq \alpha^{-1}}|v|^{2}|y|^{k^{\prime}} \rho \\
& \leq C\left(\varepsilon \alpha^{-m}+M c_{0}\left(k^{\prime}\right) \alpha^{4-m}\right) \int v_{n u l l}^{2} \rho d y
\end{aligned}
$$


where, we used the fact that $|v| \rightarrow 0$ as $s \rightarrow-\infty$ in $L^{\infty}\left(B\left(0, \alpha^{-1}\right)\right),|v(y, s)| \leq M(1+|y|)$, Lemma A.4 and $\int|v|^{2} \rho d y \leq \int v_{\text {null }}^{2} \rho d y$. We can then choose $\varepsilon$ and $\alpha$ such that for $s \leq s_{0}$, $\int|v|^{3}|y|^{m} \rho \leq \varepsilon \int v_{\text {null }}^{2}$ an we get $\mathcal{E}_{2}=o\left(v_{2}^{2}\right)$.

So finally, we have

$$
\partial_{s} v_{2}=-\frac{\beta}{\kappa} 4 v_{2}^{2}+o\left(v_{2}^{2}\right)
$$

Solving the above, we obtain

$$
v_{\text {null }}=\frac{\kappa}{4 \beta s}(1+o(1))\left(y^{2}-2\right) .
$$

This concludes the proof of Lemma A.3.

In order to finish the proof of (iii) of Proposition 2.7, we need to refine the estimates of Lemma A.3 to catch the $O\left(\log (|s|) / s^{2}\right)$. Recalling system (106) and using (113), we obtain,

$$
\dot{y} \leq-\frac{1}{2} y+c\|v(., s-s *)\|_{L_{\rho}^{2}} \leq-\frac{1}{2} y+c \frac{1}{s^{2}} .
$$

Then, integrating $\left(y e^{s / 2}\right)^{\prime} \leq C \frac{e^{s}}{s^{2}}$ between $-\infty$ and $s$, we get $y \leq \frac{C}{s^{2}}$. Doing the same for $z=\left\|v_{+}(., s)\right\|_{L_{\rho}^{2}}$, we obtain $\left(z e^{-s / 2}\right)^{\prime} \geq C \frac{e^{s}}{s^{2}}$, integrating between $s$ and $s_{0} \geq s$, we get $z \leq \frac{C}{s^{2}}$.

Proceeding as in the proof of Lemma A.3, we write

$$
\begin{aligned}
\partial_{s} v_{2} & =-\frac{\beta}{2 \kappa} \int v_{n u l l}^{2} k_{2}(y) \rho(y)+\frac{\beta}{2 \kappa} \int\left(v_{\text {null }}^{2}-v^{2}\right) k_{2}(y) \rho(y)+\int g(v) k_{2}(y) \rho(y), \\
& =-\frac{\beta}{2 \kappa} 8 v_{2}^{2}+\frac{\beta}{2 \kappa} \mathcal{E}_{1}+\mathcal{E}_{2} .
\end{aligned}
$$

Then, we have

$$
\begin{aligned}
\left|\mathcal{E}_{1}\right| & \leq \int\left|v_{+}+v_{-}\right| \times\left|v+v_{\text {null }}\right|\left|k_{2}(y)\right| \rho, \\
& \leq c\left(\int\left|v_{+}+v_{-}\right|^{2} \rho\right)^{1 / 2}\left\{\left(v^{2} k_{2}^{2}(y) \rho\right)^{1 / 2}+\left(\int v_{\text {null }}^{2} k_{2}^{2} \rho\right)^{1 / 2}\right\}, \\
& \leq \varepsilon\left(\int v_{\text {null }}^{2} \rho\right)^{1 / 2}\left\{c\left(\int v^{4} \rho\right)^{1 / 4}+c\left(\int v_{\text {null }}^{2} \rho\right)^{1 / 2}\right\} .
\end{aligned}
$$

Using the fact that $\left\|v_{\text {null }}(., s)\right\|_{L_{\rho}^{2}} \sim \frac{C}{s}$ and (113), we have

$$
\int v^{4} \rho \leq c\left(v^{2}(., s-s *) \rho\right)^{2} \leq \frac{c}{(s-s *)^{2}} \leq \frac{c}{s^{2}} .
$$

Thus, $\mathcal{E}_{1} \leq \frac{C}{s^{3}}$. Similarly, we obtain $\mathcal{E}_{2} \leq \frac{C}{s^{3}}$. Then, we have from (117):

$$
\partial_{s} v_{2}=-\frac{4 \beta}{\kappa} v_{2}^{2}+O\left(\frac{1}{s^{3}}\right)=-\frac{4 \beta}{\kappa} v_{2}^{2}\left(1+O\left(\frac{1}{s}\right)\right) .
$$

By integerating, we obtain the desired estimation

$$
v_{2}(s)=\frac{\kappa}{4 \beta s}+O\left(\log (|s|) / s^{2}\right) .
$$

This concludes the proof of Proposition 2.7. 


\section{B Equations of $Z$ in Parts 4 and 5}

Equation of $Z$ in Part 4: In this part we establish the equations (67) and (72) satisfied by $Z$ and finishes the proof of Lemma 2.14 and Claim 2.16. If we recall from (66) that $\nu=w-F$, where $F$ is defined by (62), then we see from (6) that $\nu$ satisfies the following equation for all $(y, s) \in \mathbb{R} \times \mathbb{R}$, such that for $|y|<4 \varepsilon_{0} \sqrt{-s}$.

$$
\partial_{s} \nu=\mathcal{L} \nu+l(y, s) \nu-B(\nu)+R(y, s),
$$

where $\mathcal{L}$ is defined in (48),

$$
\begin{aligned}
& l(y, s)=\left(-\frac{\beta}{\beta+1}+\beta F^{-(\beta+1)}\right) \\
& B(\nu)=(F+\nu)^{-\beta}-F^{-\beta}+\beta F^{-(\beta+1)} \nu, \\
& R(y, s)=-\partial_{s} F+\partial_{y}^{2} F-\frac{1}{2} y \cdot \partial_{y} F+\frac{F}{\beta+1}-F^{-\beta} .
\end{aligned}
$$

Using Taylor's formula, the fact that $F$ is bounded for $|y| \leq 4 \varepsilon_{0} \sqrt{-s}$ and proceeding as in the proof of Lemma 2.6, we readily obtain for all $s \leq s_{0}$ and $|y|<4 \varepsilon_{0} \sqrt{-s}$

$$
|B(\nu)| \leq C|\nu|^{2}, \quad|R(y, s)| \leq C\left(\frac{|y|^{2}+1}{s^{2}}+\chi_{\varepsilon_{0}}\right), \quad|l(y, s)| \leq C \min \left[\frac{\left(1+\left|y^{2}\right|\right)}{|s|}, 1\right]
$$

with $\chi_{\varepsilon_{0}}$ defined in (64). Therefore, we write for $|s|$ large enough and $|y| \leq 4 \varepsilon_{0} \sqrt{-s}$ :

$$
|l(y, s)| \leq C\left\{\frac{\left(1+\varepsilon_{0}^{2}|s|\right)}{|s|}+\chi_{\varepsilon_{0}}\right\} \leq C\left\{2 \varepsilon_{0}^{2}+\chi_{\varepsilon_{0}}\right\} .
$$

Using Kato's inequality, we obtain for $z=|\nu|,|s|$ large enough and $|y| \leq 4 \varepsilon_{0} \sqrt{-s}$ :

$$
\partial_{s} z-\partial_{y}^{2} z+\frac{1}{2} y \cdot \partial_{y} z-(1+\sigma) z \leq C\left(z^{2}+\frac{\left(y^{2}+1\right)}{s^{2}}+\chi_{\varepsilon_{0}}\right)
$$

where we fix $\varepsilon_{0}$ small enough so that $\sigma=C \varepsilon_{0}^{2} \leq \frac{1}{100}$ (use the fact that $\chi_{\varepsilon_{0}} \gamma \leq \gamma^{2}+\chi_{\varepsilon_{0}}$ ), with the cut-off function $\gamma$ defined by (65).

We define $Z=z \gamma$ and we obtain for $|s|$ large enough:

$$
\begin{aligned}
\partial_{s} Z-\partial_{y}^{2} Z+\frac{1}{2} y \cdot \partial_{y} Z-(1+\sigma) Z & \leq C\left(Z^{2}+\frac{\left(y^{2}+1\right)}{s^{2}}+\chi_{\varepsilon_{0}}\right) \\
& +z\left(\partial_{s} \gamma-\partial_{y}^{2} \gamma+\frac{y}{2} \cdot \partial_{y} \gamma\right)-2 \partial_{y} \gamma \cdot \partial_{y} z
\end{aligned}
$$

(here, we used the fact that $\left.\gamma z^{2}=Z^{2}+\left(\gamma-\gamma^{2}\right) z^{2} \leq Z^{2}+C \chi_{\varepsilon_{0}}\right)$. The last terms in this equation are the cut-off terms. By (i) of Lemma 2.1 and (37), we get

$$
z(y, s) \leq C(1+\sqrt{-s}) \text { for all }|y| \leq 4 \varepsilon_{0} \sqrt{-s},
$$

then we obtain using the fact that $z\left(\partial_{s} \gamma-\partial_{y}^{2} \gamma+\frac{y}{2} \cdot \partial_{y} \gamma\right)-2 \partial_{y} \gamma \partial_{y} z \leq C(1+\sqrt{-s}) \chi_{\varepsilon_{0}}-$ $2 \operatorname{div}\left(z \partial_{y} \gamma\right)$, we obtain for $|s|$ large enough:

$\partial_{s} Z-\partial_{y}^{2} Z+\frac{1}{2} y \cdot \partial_{y} Z-(1+\sigma) Z \leq C\left(Z^{2}+\frac{\left(y^{2}+1\right)}{s^{2}}+(1+\sqrt{-s}) \chi_{\varepsilon_{0}}\right)-2 \operatorname{div}\left(|\nu| \partial_{y} \gamma\right)$ 
which is the desired equation (67) in Lemma 2.14.

In the following, we will establish inequality (72). Let us rewrite (118) as follow

$$
\partial_{s} \nu=\left(\mathcal{L}-\frac{\beta}{\beta+1}\right) \nu-B_{1}(\nu)+R(y, s),
$$

where

where $B_{1}(\nu)=(F+\nu)^{-\beta}-F^{-\beta}$ and $R$ is defined in (119)

Proceeding as in the proof of Lemma 2.6, we get $|B(\nu)| \leq C|\nu|$. Now, we do as in the proof of (67), and we obtain the wanted result (72).

Equation of $Z$ in Part 5: In the following, we determine the equations satisfied by $Z$ in Part 5. We note by $\nu=w-f$. We can see from (82), that $\nu$ satisfies the following equation for all $(y, s) \in \mathbb{R} \times \mathbb{R}$, such that for $|y|<4 \varepsilon_{0} e^{-s / 2}$

$$
\partial_{s} \nu=\mathcal{L} \nu+l(\nu)-B(\nu)+R(y, s),
$$

where

$$
\begin{aligned}
& l(\nu)=\left(-\frac{\beta}{\beta+1}+\beta f^{-(\beta+1)}\right) \nu=l(y, s) \nu, \\
& B(\nu)=\left((f+\nu)^{-(\beta)}-f^{-\beta}+\beta f^{-(\beta+1)}\right) \nu, \\
& R(y, s)=e^{s} \partial_{y}^{2} G\left(y e^{s / 2}\right) .
\end{aligned}
$$

Using Taylor's formula, the fact that $f$ is bounded for $|y| \leq 4 \varepsilon_{0} e^{s / 2}$ and proceeding as in the proof of Lemma 2.6, we prove that for $|s|$ large and $|y| \leq 4 \varepsilon_{0} e^{-s / 2}$

$$
|B(\nu)| \leq C|\nu|^{2},|R(y, s)| \leq C e^{s}+\chi_{\varepsilon_{0}}(y, s),
$$

with $\chi_{\varepsilon_{0}}$ is defined by (85). We obtain for $|y| e^{s / 2} \leq 4 \varepsilon_{0}$ and $s$ large

$$
|l(y, s)| \leq C \min \left[|y| e^{s / 2}, 1\right] .
$$

If we consider $\chi_{\varepsilon_{0}}$ defined in (85), then, we write for $|s|$ large and $|y| \leq 4 \varepsilon_{0} e^{-s / 2}$ :

$$
|l| \leq C\left\{|y| e^{s / 2}+\chi_{\varepsilon_{0}}\right\} \leq C\left\{\varepsilon_{0}+\chi_{\varepsilon_{0}}\right\} .
$$

Using Kato's inequality, we obtain for $z=|\nu|,|s|$ large enough and $|y| e^{s} \leq 4 \varepsilon_{0}$,

$$
\partial_{s} z-\partial_{y}^{2} z+\frac{1}{2} y \cdot \partial_{y} z-(1+\sigma) z \leq C\left(z^{2}+e^{s}+\chi_{\varepsilon_{0}}\right)
$$

where $\sigma=C \varepsilon_{0}=\frac{1}{100}$. Now, we consider the cut-off function $\gamma$, we define $Z=z \gamma$ and we obtain for $|s|$ large:

$$
\begin{aligned}
& \partial_{s} Z-\partial_{y}^{2} Z+\frac{1}{2} y \cdot \partial_{y} Z-(1+\sigma) Z \\
& \leq C\left(Z^{2}+e^{s}+\chi_{\varepsilon_{0}}\right)-z\left(\partial_{s} \gamma-\partial_{y}^{2} \gamma+\frac{y}{2} \cdot \partial_{y} \gamma\right)+2 \partial_{y} \gamma \partial_{y} z
\end{aligned}
$$

The last terms in this equation are the cut-off terms. By (i) of Lemma 2.1 we have $|\nu| \leq C\left(1+e^{-s / 2}\right)$.Then, using $z\left(\partial_{s} \gamma-\partial_{y}^{2} \gamma+\frac{y}{2} \cdot \partial_{y} \gamma\right)-2 \partial_{y} \gamma \partial_{y} z \leq C \chi_{\varepsilon_{0}}+2 \operatorname{div}\left(z \partial_{y} \gamma\right)$, we obtain for $|s|$ large:

$$
\partial_{s} Z-\partial_{y}^{2} Z+\frac{1}{2} y \cdot \partial_{y} Z-(1+\sigma) Z \leq C\left(Z^{2}+e^{s}+\left(1+e^{-s / 2}\right) \chi_{\varepsilon_{0}}\right)-2 \operatorname{div}\left(\left(|\nu| \partial_{y} \gamma\right),\right.
$$

which is the desired equation in (84). 


\section{References}

[CHO98] S. J. Chapman, B. J. Hunton, and J. R. Ockendon. Vortices and boundaries. Quart. Appl. Math., 56(3):507-519, 1998.

[DL89] K. Deng and H.A. Levine. On the blow up of $u_{t}$ at quenching. Proc. Amer. Math. Soc., 106(4):1049-1056, 1989.

[DM05] J. Dávila and M. Montenegro. Existence and asymptotic behavior for a singular parabolic equation. Trans. Amer. Math. Soc., 357(5):1801-1828 (electronic), 2005.

[FG93] S. Filippas and J.S. Guo. Quenching profiles for one-dimensional semilinear heat equations. Quart. Appl. Math., 51(4):713-729, 1993.

[FHQ92] M. Fila, J. Hulshof, and P. Quittner. The quenching problem on the $N$ dimensional ball. 7:183-196, 1992.

[FK92a] M. Fila and B. Kawohl. Asymptotic analysis of quenching problems. Rocky Mountain J. Math., 22(2):563-577, 1992.

[FK92b] S. Filippas and R.V. Kohn. Refined asymptotics for the blowup of $u_{t}-\Delta u=$ uSp p. Comm. Pure Appl. Math., 45(7):821-869, 1992.

[FKBL92] M. Fila, Kawohl, B., and H. A. Levine. Quenching for quasilinear equations. Comm. Partial Differential Equations, 17(3-4):593-614, 1992.

[GK85] Y. Giga and R.V. Kohn. Asymptotically self-similar blow-up of semilinear heat equations. Comm. Pure Appl. Math., 38(3):297-319, 1985.

[GL07] S. Gaucel and M. Langlais. Some remarks on a singular reaction-diffusion system arising in predator-prey modeling. Discrete Contin. Dyn. Syst. Ser. B, 8(1):61-72 (electronic), 2007.

[Guo90] J.S. Guo. On the quenching behavior of the solution of a semilinear parabolic equation. J. Math. Anal. Appl., 151(1):58-79, 1990.

[Guo91a] J.S. Guo. On the quenching rate estimate. Quart. Appl. Math., 49(4):747-752, 1991.

[Guo91b] J.S. Guo. On the semilinear elliptic equation $\Delta w-\frac{1}{2} y \cdot \nabla w+\lambda w-w \mathrm{Sp}-\beta=0$ in RSpn. Chinese J. Math., 19(4):355-377, 1991.

[GV02] V.A. Galaktionov and J. L. Vázquez. The problem of blow-up in nonlinear parabolic equations. Discrete Contin. Dyn. Syst., 8(2):399-433, 2002. Current developments in partial differential equations (Temuco, 1999).

[HV93] M. A. Herrero and J. J. L. Velázquez. Blow-up behaviour of one-dimensional semilinear parabolic equations. Ann. Inst. H. Poincaré Anal. Non Linéaire, 10(2):131-189, 1993. 
[Kaw75] H. Kawarada. On solutions of initial-boundary problem for $u_{t}=u_{x x}+1 /(1-u)$. Publ. Res. Inst. Math. Sci., 10(3):729-736, 1974/75.

[Lev93] H. A. Levine. Quenching and beyond: a survey of recent results. In Nonlinear mathematical problems in industry, II (Iwaki, 1992), volume 2 of GAKUTO Internat. Ser. Math. Sci. Appl., pages 501-512. Gakkōtosho, Tokyo, 1993.

[LLT07] K. W. Liang, P. Lin, and R. C. E. Tan. Numerical solution of quenching problems using mesh-dependent variable temporal steps. Appl. Numer. Math., 57(5-7):791-800, 2007.

[MM00] Y. Martel and F. Merle. A Liouville theorem for the critical generalized Korteweg-de Vries equation. J. Math. Pures Appl. (9), 79(4):339-425, 2000.

[MZ97] F. Merle and H. Zaag. Reconnection of vortex with the boundary and finite time quenching. Nonlinearity, 10(6):1497-1550, 1997.

[MZ98a] F. Merle and H. Zaag. Optimal estimates for blowup rate and behavior for nonlinear heat equations. Comm. Pure Appl. Math., 51(2):139-196, 1998.

[MZ98b] F. Merle and H. Zaag. Refined uniform estimates at blow-up and applications for nonlinear heat equations. Geom. Funct. Anal., 8(6):1043-1085, 1998.

[MZ00] F. Merle and H. Zaag. A Liouville theorem for vector-valued nonlinear heat equations and applications. Math. Ann., 316(1):103-137, 2000.

[MZ08] F. Merle and H. Zaag. Openness of the set of non characteristic points and regularity of the blow-up curve for the $1 \mathrm{~d}$ semilinear wave equation. Comm. Math. Phys., 2008. to appear.

[NZ10] N. Nouaili and H. Zaag. A liouville theorem for vector valued semilinear heat equations with no gradient structure and applications to blow-up. Trans. Amer. Math. Soc., 2010. to appear.

[Sal04] T. Salin. Quenching-rate estimate for a reaction diffusion equation with weakly singular reaction term. Dyn. Contin. Discrete Impuls. Syst. Ser. A Math. Anal., 11(4):469-480, 2004.

[Vel92] J. J. L. Velázquez. Higher-dimensional blow up for semilinear parabolic equations. Comm. Partial Differential Equations, 17(9-10):1567-1596, 1992.

[Vel93] J. J. L. Velázquez. Classification of singularities for blowing up solutions in higher dimensions. Trans. Amer. Math. Soc., 338(1):441-464, 1993.

[Zaa01] H. Zaag. A Liouville theorem and blowup behavior for a vector-valued nonlinear heat equation with no gradient structure. Comm. Pure Appl. Math., 54(1):107$133,2001$.

Nejla Nouaili

Université Paris Dauphine, CEREMADE.

Place du maréchal de Lattre de Tassigny 
75016 Paris, France

email: nouaili@ceremade.dauphine.fr 